\title{
ARTEMIS Science Objectives
}

\author{
D.G. Sibeck • V. Angelopoulos • D.A. Brain • G.T. Delory • J.P. Eastwood • \\ W.M. Farrell · R.E. Grimm · J.S. Halekas • H. Hasegawa · P. Hellinger • \\ K.K. Khurana · R.J. Lillis · M. Øieroset • T.-D. Phan · J. Raeder • C.T. Russell • \\ D. Schriver $\cdot$ J.A. Slavin $\cdot$ P.M. Travnicek $\cdot$ J.M. Weygand
}

Received: 25 July 2010 / Accepted: 8 April 2011

(C) US Government 2011

\begin{abstract}
NASA's two spacecraft ARTEMIS mission will address both heliospheric and planetary research questions, first while in orbit about the Earth with the Moon and subsequently while in orbit about the Moon. Heliospheric topics include the structure of the Earth's magnetotail; reconnection, particle acceleration, and turbulence in the Earth's magnetosphere, at the bow shock, and in the solar wind; and the formation and structure of the
\end{abstract}

D.G. Sibeck $(\bowtie)$

Code 674, GSFC/NASA, Greenbelt, MD 20771, USA

e-mail: david.g.sibeck@nasa.gov

V. Angelopoulos

IGPP, UCLA, Los Angeles, CA 90095, USA

D.A. Brain · G.T. Delory · J.S. Halekas · R.J. Lillis · M. Øieroset · T.-D. Phan

UCB, Berkeley, CA, USA

J.P. Eastwood

Imperial College, London, UK

W.M. Farrell · J.A. Slavin

NASA/GSFC, Greenbelt, MD, USA

R.E. Grimm

SWRI, Boulder, CO, USA

H. Hasegawa

ISAS, Sagamihara, Japan

P. Hellinger · P.M. Travnicek

Astronomical Institute, Prague, Czech Republic

K.K. Khurana - C.T. Russell · D. Schriver · P.M. Travnicek · J.M. Weygand

UCLA, Los Angeles, CA, USA

J. Raeder

UNH, Durham, NH, USA

Published online: 17 May 2011 
lunar wake. Planetary topics include the lunar exosphere and its relationship to the composition of the lunar surface, the effects of electric fields on dust in the exosphere, internal structure of the Moon, and the lunar crustal magnetic field. This paper describes the expected contributions of ARTEMIS to these baseline scientific objectives.

Keywords ARTEMIS · Moon · Reconnection · Particle acceleration · Turbulence · Wake · Lunar surface $\cdot$ Lunar core $\cdot$ Dust $\cdot$ Electric fields $\cdot$ Crustal anomalies

\section{Introduction}

ARTEMIS (Acceleration, Reconnection, Turbulence, and Electrodynamics of the Moon's Interaction with the Sun) is NASA's first dual spacecraft mission to the Moon. By mission design and both efficient navigation and flight operations during the primary phase of the THEMIS mission from February 2007 to September 2009 (Angelopoulos 2008), the outermost two THEMIS spacecraft P1 and P2 found themselves with fuel reserves sufficient to undertake a series of over forty maneuvers, including multiple lunar approaches and flybys, that enabled them to reach and parallel the Moon during trans-lunar injection phases from October 2009 through September 2010. From October 2010 to January 2011, the two spacecraft were in Lissajous orbits at the Lagrange points of the Earth-Moon system that straddled the Moon and were separated by distances on the order of $\sim 20$ Earth radii $\left(R_{\mathrm{E}}\right)$. From January to July 2011, their Lissajous orbits were separated by 5 to $20 R_{\mathrm{E}}$ on the same side of the Moon. In July 2011 they entered lunar orbits (one prograde, the other retrograde) with separation distances ranging from $500 \mathrm{~km}$ to 20 lunar radii $\left(R_{\mathrm{L}}\right)$. Note that $1 R_{\mathrm{E}}$ is $6371 \mathrm{~km}$, while $1 R_{\mathrm{L}}$ is $1737 \mathrm{~km}$.

Observations from lunar orbit over a such wide range of interspacecraft separations are ideal for addressing a number of longstanding heliophysics objectives, including defining the characteristics of reconnection and turbulence in the solar wind and distant magnetotail, the nature of particle acceleration at the Earth's bow shock and interplanetary discontinuities, and the electrodynamics of the solar wind's interaction with the Moon. However, by careful optimization of mission parameters, in particular periselene and orbital inclination, the fully-instrumented and fully-functional ARTEMIS spacecraft can also be employed to address a set of planetary research objectives including the composition and magnetization of the lunar surface, the effects of electric fields on dust in the vicinity of the Moon, and the structure of the lunar interior. With orbits that will remain stable for over a decade, ARTEMIS will provide observations of the lunar environment over solar cycle time scales, offer measurements that overlap and enhance those of NASA's Lunar Reconnaissance Orbiter (LRO) and Lunar Atmosphere and Dust Environment Explorer (LADEE) missions, and provide crucial solar wind observations for correlative studies with other NASA missions to the Earth's magnetosphere and ionosphere/thermosphere.

Angelopoulos (2011, this issue) provides a mission overview for ARTEMIS that summarizes the overall scientific objectives, orbits, instrumentation, mission and science operations that are expanded upon in individual papers in this compendium. This paper describes how the above mission elements will enable the ARTEMIS team to reach scientific closure. In terms of organization, Sect. 2 describes ARTEMIS planetary objectives while Sect. 3 describes the ARTEMIS heliophysics objectives. Section 4 presents conclusions. 


\section{ARTEMIS Planetary Science Objectives}

The Moon holds critical information regarding the origin of the solar system. Because it is devoid of plate tectonics, volcanism and surface-altering atmospheric processes, the Moon's surface has recorded the 4.5 billion years of solar system history more purely than any other planetary body. Understanding the lunar surface and the stratification of the lunar interior provides a window into the early history of the Earth-Moon system and can shed considerable light on the evolution of terrestrial planets such as Mars and Venus. The surface is constantly sputtering ions and dust, which then enter and circulate within the lunar exosphere before escaping into the solar wind. Ions 'picked-up' by the solar wind gain similar velocities but mass-dependent energies as they follow mass-dependent trajectories. ARTEMIS will use its energy-angle spectroscopic capability and its electric and magnetic field sensors to determine the motion, source, composition, and flux of exospheric ions. The surface electric fields that loft and transport dust particles can be remotely sensed using electron reflectometry or measured directly from altitude. ARTEMIS will use its electromagnetic field measurements to determine the forces acting on dust populations and cause their acceleration and deposition or loss in the lunar environment.

Apollo-era data indicates that the Moon formed via the impact of a Mars-sized object with the early Earth, and later differentiated into primary crust, mantle residuum, and possibly a small iron-rich core. Radial profiles for the Moon's temperature and composition, and their lateral variability today, hold important clues regarding the history of lunar differentiation. Broadband ( $\ll 10 \mathrm{mHz}$ to $10 \mathrm{~Hz}$ ) electromagnetic sounding will improve our knowledge of these state variables for the core, mantle, and crust. The sensitive magnetometers on ARTEMIS will approach the Moon on orbits that bring them, one at a time, to within $100 \mathrm{~km}$ of the lunar surface, i.e. to altitudes close enough to detect the core response to varying external drivers but distant enough to minimize perturbations from crustal anomalies. ARTEMIS also provides sensitive horizontal electric field measurements from $1-10 \mathrm{~Hz}$ that will further enable magnetotelluric investigations.

Finally, ARTEMIS will use its comprehensive particle and field sensors to study the interaction of the solar wind with lunar crustal magnetic anomalies. Initial Kaguya observations have already provided significant new information on an ion sheath, electron heating and solar wind reflection around magnetic anomalies at $100 \mathrm{~km}$ (Saito et al. 2010), but wave properties and solar wind particle flow around the strong field region remain poorly understood. ARTEMIS will study the magnetic anomalies to infer properties of the ancient, seed magnetic field and to determine the accessibility of the solar wind to the surface and the effect it has on lunar surface ageing. Electric field and plasma wave data, together with ion and electron measurements in the vicinity of the mini-magnetosphere and shock-like structures (e.g., Halekas et al. 2006a) that form around the crustal anomaly (the first comprehensive plasma measurements attempted at the Moon) promise exciting new science with possibly significant ramifications for planetary evolution.

\subsection{Exospheric Ions and Plasma Pick-up}

Forty years after the first Apollo landings, the composition and structure of the lunar exosphere remain poorly understood. A number of studies have used ground-based measurements to investigate the existence, extent, variability and likely sources of easily observed exospheric species such as sodium and potassium (Potter and Morgan 1988; Tyler et al. 1988; Mendillo et al. 1991, 1999; Wilson et al. 2003, 2006). However, the behavior of these species may not be representative of other, more abundant, species (Potter and Morgan 1988; Flynn and Mendillo 1993). 

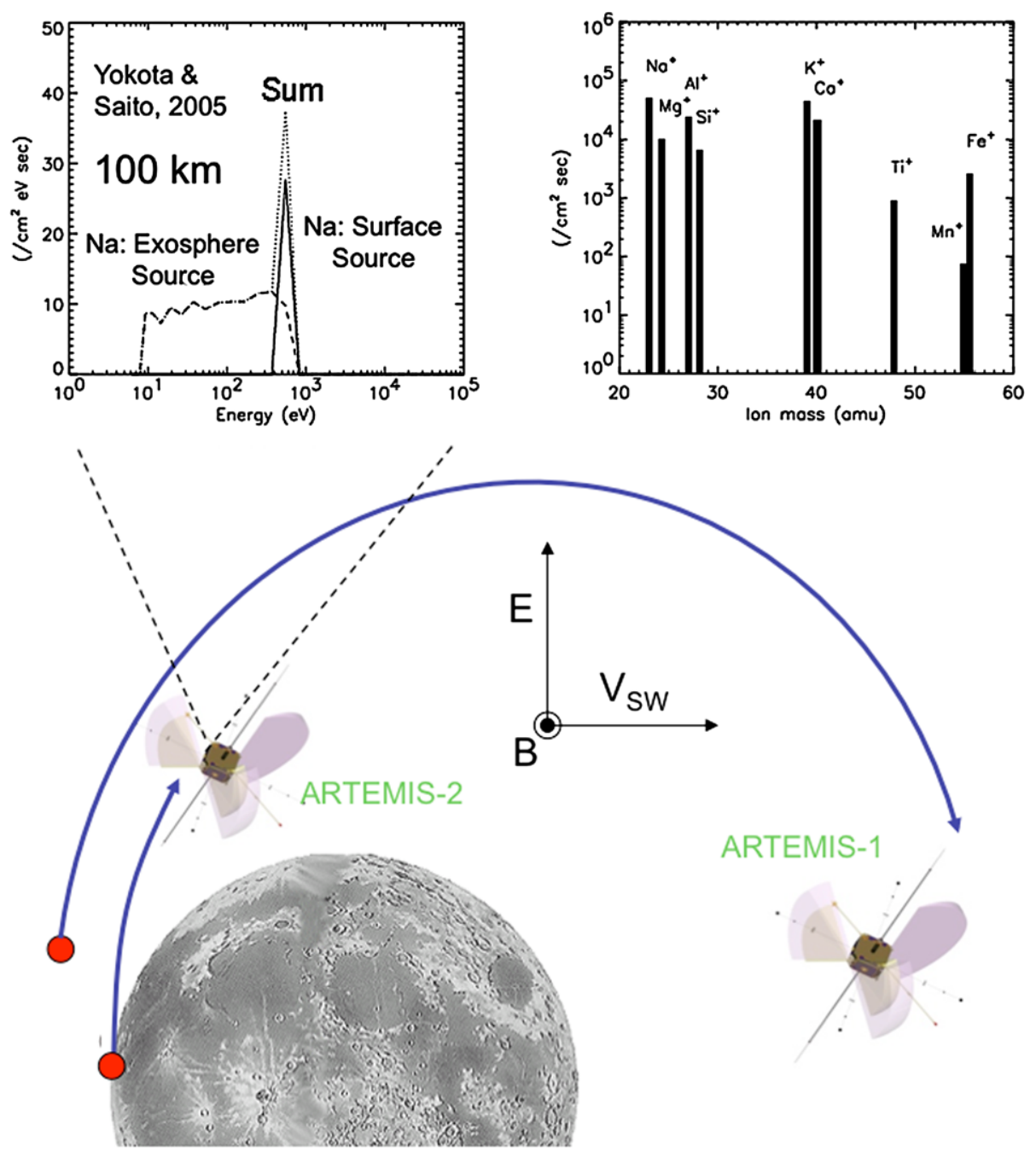

Fig. 1 A schematic showing the trajectories of recently picked-up ions and the expected measurements of the fluxes (top left) and composition (top right) of the picked-up ions

In situ measurements of pickup ions offer an appealing complementary means of probing surface and exospheric properties and processes at the Moon (e.g. Hartle and Thomas 1974; Cladis et al. 1994; Yokota and Saito 2005; Hartle and Killen 2006; Hartle and Sittler 2007; Saito et al. 2010). Newly created ions, produced by solar wind sputtering, photostimulated desorption, micro-meteoriod impact vaporization or ionization of exospheric gases are generated at relatively low energies $(0.01-10 \mathrm{eV})$, but immediately feel the effect of solar wind magnetic and electric field (Stern 1999; Wurtz et al. 2007). The 'picked-up' ions are then accelerated in cycloidal trajectories like that shown in Fig. 1.

In situ measurements by ion instruments on the space plasma missions AMPTE, WIND, and GEOTAIL confirmed the presence of lunar heavy ions far downstream from the Moon in the solar wind and in the distant magnetotail (Hilchenbach et al. 1991, 1993; Kirsch et al. 1997; Mall et al. 1998), but relied on fortuitous configurations of the solar wind and 
IMF and were not able to provide comprehensive information on exospheric composition, source rates, or variability. Further, these studies could not distinguish between lunar ions originating at the surface and those created in the exosphere.

More recent studies have shown that it is possible to separate the signatures of surface and exospheric ions, using measurements made at altitudes smaller than the typical gyroradius of newly ionized lunar species. For example, the upper left inset in Fig. 1 shows the result of a computation by Yokota and Saito (2005) of the expected differential fluxes of $\mathrm{Na}$ at $\sim 100 \mathrm{~km}$ altitudes resulting from both surface sputtering and exospheric photoionization. The surface ions are nearly monoenergetic, while the atmospheric ions spread out in energy due to the extended source and low observation altitude. At higher altitudes, the ions would be accelerated still further, resulting in a much more monoenergetic spectrum and much higher ion energies for both surface and exospheric sources (with little reduction in flux unless significant scattering occurs in one ion gyroperiod). At distances within two gyroradii from the source, one may discriminate between ions with different masses by comparing observed energies and gyrophases with those predicted for the cyclical motion shown in Fig. 1, though distinguishing between surface and exospheric ions becomes more difficult.

Kaguya findings have recently advanced our understanding of the solar wind's interaction with the lunar surface as well as the near-Moon wake. Kaguya's in situ ion mass spectrometry was used to confirm the presence of $\mathrm{Na}^{+}$in addition to $\mathrm{He}^{+}, \mathrm{C}^{+}, \mathrm{O}^{+}, \mathrm{K}^{+}$, and $\mathrm{Ar}^{+}$in the sunlit lunar exosphere at altitudes $\sim 100 \mathrm{~km}$ when the Moon was in the solar wind (Yokota et al. 2009) and Earth's magnetosphere (Tanaka et al. 2009). Some ions originated from the exosphere, others from the surface. Other species may be present as well. The heavy ion flux varies with solar zenith angle but not with solar wind flux or meteor shower occurrences, suggesting a stable driver for the sputtering process.

Kaguya observations also indicate that from 0.1 to $1 \%$ of the solar wind protons incident upon the Moon are reflected. Solar wind convection electric fields accelerate some of these protons to speeds triple those of the solar wind (Saito et al. 2008). The pickup ions find access not only to the high latitude wake, but also areas deep within the low latitude and low altitude wake through fully kinetic processes. Knowledge of the solar wind electric and magnetic fields suffices to reconstruct observed proton spectra from dayside reflected proton sources.

The ESA and SST instruments on ARTEMIS will measure the energy and direction of pickup ion particle distributions, while the EFI and FGM instruments will measure the ambient electric and magnetic fields. With this information, researchers will be able to backtrack observed pickup ions to either surface or exospheric sources. Furthermore, they will be able to roughly determine the ion mass, since both the ion energy and the size of the cycloidal trajectories scale with mass. ARTEMIS can therefore use pickup ion measurements to remotely probe the properties of the surface and the exosphere. Measurements from the ARTEMIS probe measuring the lunar exosphere can then be combined with observations from the other ARTEMIS probe to determine the response of the exosphere to solar wind drivers.

Although ARTEMIS will generally be further away from the Moon than Kaguya, the large geometric factor of the ESA total ion instruments (a factor of $\sim 10$ greater than those on Kaguya) will enable sensitive measurements of the pickup ions under stable solar wind conditions over distances ranging from $100 \mathrm{~km}$ to several thousand $\mathrm{km}$ from the lunar surface. When applied as a function of lunar phase the technique will determine the dependence of the lunar exosphere on lunar longitude undergoing illumination, thereby providing the ion composition versus selenographic longitude. Since the pickup ion trajectories measured by ARTEMIS can be back-traced, the latitudinal exospheric ion distribution can also be determined. 
A second method of indirect detection of lunar ions may also be possible using ARTEMIS measurements. Pickup ions form ring distributions that can generate electromagnetic ion cyclotron waves. It may be possible to detect these waves using the ARTEMIS vector magnetic field data. Waves from pickup ions have been identified in the plasma environments of many solar system bodies, including Venus, Mars, and even the moons of the giant planets (Russell et al. 1990; Kivelson et al. 1996; Paterson et al. 1999; Grebowsky et al. 2004; Dougherty et al. 2006; Russell and Blanco-Cano 2007; Delva et al. 2008), and their presence or absence can be used to constrain the local density of pickup ions, and therefore the source strength.

Finally, synergistic measurements made by ARTEMIS and other orbiting spacecraft can be used to constrain exospheric properties. The Lunar Reconnaissance Orbiter (LRO) mission carries the Lyman-Alpha Mapping Project (LAMP) ultraviolet instrument (Gladstone et al. 2010), while the upcoming Lunar Atmosphere Dust Environment Explorer (LADEE) mission carries the NMS for neutral mass spectrometry and the UVS ultraviolet/visual spectrometer (Delory et al. 2009). These missions thus measure gases before ionization, while ARTEMIS measures them post-ionization. By coordinating ARTEMIS measurements with those from these and other spacecraft, we can greatly advance our understanding of the lunar exosphere and its coupling to the surface and the space environment.

\subsection{Surface Charging, Electric Fields, and Dust}

Although one might think of the lunar environment as essentially dormant, it is in fact very active electrically. Since the Moon has only a tenuous exosphere and no global magnetic field, its surface lies directly exposed to the impact of solar UV and X-rays as well as solar wind plasma and energetic particles. This creates a complex and dynamic lunar electric environment, with the surface typically charging positive in sunlight and negative in shadow to potentials that vary over orders of magnitude in response to changing solar illumination and plasma conditions. These potentials have been measured by instruments on the surface and by Lunar Prospector in orbit, but we still do not fully understand the near-surface environment, especially the role of secondary and photoemitted electrons, and the structure of the plasma sheath at the surface.

The largest observed lunar potentials typically occur on the nightside, in the absence of photoemission, where ambient plasma currents primarily drive surface charging (Manka 1973; Stubbs et al. 2007). Using measurements of the angular distribution of reflected electrons and accelerated secondary electrons from the surface, Lunar Prospector provided estimates of negative nightside lunar surface potentials of $\sim-100 \mathrm{~V}$ or less in the wake and magnetospheric tail lobes (Halekas et al. 2002a, 2008c), and occasionally as high as $-2-4 \mathrm{kV}$ in the magnetospheric plasmasheet (Halekas et al. 2005, 2008c) and during SEP events (Halekas et al. 2007). However, all Lunar Prospector measurements were fundamentally handicapped by a lack of spacecraft potential measurements and the spectrometer's rather coarse electron energy resolution. ARTEMIS's high resolution full plasma and electric field measurements will enable researchers to measure lunar surface potentials from orbit accurately and precisely, as well as determine their response to external influences. The excellent energy resolution of the electron spectrometers on ARTEMIS will also enable a better understanding of the importance of lunar photoelectrons and secondary electrons, and may allow us to determine whether the potential in the plasma sheath varies monotonically, or non-monotonically as suggested by recent simulations (Poppe and Horanyi 2010).

Near-surface electric fields like those described above can loft micron-sized charged dust grains (Nitter et al. 1998). The horizon 'glow' observed by cameras on the landed Surveyors 
5, 6 and 7 at lunar twilight was attributed to sunlight scattering by dust electrostaticallylevitated just above the lunar surface by electric fields at the terminators (Criswell 1973; Rennilson and Criswell 1974). Astronauts in Apollo's command module also witnessed a twilight horizon glow extending 10's of kilometers above the lunar surface which was suggested to result from surface ejection of submicron grains to high altitudes (McCoy and Criswell 1974; McCoy 1976). Lunar Prospector has confirmed the presence of strong and dynamic near-surface electric fields that are capable of lofting small charged grains to high altitudes (10's of km) in a dust 'fountain' effect (Stubbs et al. 2006). These near surface electric fields may be strongest at the terminator-the starting location of the lunar wake (Farrell et al. 2007).

The nature of the wake plasma discontinuity at the terminator remains unknown. Ideally, there should be a 'perfect' plasma discontinuity at the terminator dividing flowing solar wind plasma from a perfect plasma void. Due to their higher thermal velocities, electrons should migrate into the plasma void further than ions, thereby creating a very large ambipolar E-field just above the lunar surface. This initial plasma expansion E-field could drive dust transport at the terminator (Farrell et al. 2007). However, plasma discontinuities tend to dissipate by radiating plasma waves at group velocities that exceed the speed of the discontinuity. These oscillatory waves and their related ULF/VLF electric fields may diminish the ambipolar effect and associated dust lofting.

ARTEMIS will examine the wake flank/discontinuity as a function of distance from the terminator, providing enough passes under varying solar wind and IMF directions to provide a unique evaluation of the effectiveness of the ambipolar forces in surface dust lofting. In essence, ARTEMIS will cross the discontinuity at various stages of its evolution, providing detailed observations of the plasma expansion and discontinuity dissipation processes. For similar solar wind and IMF configurations, the wake flank can be considered time stationary, and passages through the flank at various altitudes are equivalent to examining the discontinuity dissipation process as a function of time. The initial plasma expansion process is not only fundamentally new science, but also crucial to understanding surface dust lofting from E-fields at the terminator.

\subsection{Internal Structure}

Electromagnetic sounding encompasses a wide variety of inductive methods used to sense the interior of the Earth, the Moon and other planetary bodies (Schuster and Lamb 1889; Grant and West 1965; Sonett 1982; Khurana et al. 1998; Simpson and Bahr 2005). Suitable signals for planetary-scale soundings arise from the solar wind, magnetospheres, ionospheres, and lightning, depending on the planetary environment (Grimm 2009). These timevarying electromagnetic source fields induce eddy currents in planetary interiors, whose secondary electromagnetic fields are detected at the surface. The secondary fields shield the interior according to the skin-depth effect, which is exploited by electromagnetic sounding by using impedance measurements over a range of frequency to reconstruct conductivity over a range of depth.

Electromagnetic induction studies performed during Apollo gave an indication of the deep electrical conductivity profile and limited the radius of any strongly conducting core to less than about $20 \%$ of the lunar radius (e.g, Sonett et al. 1972; Dyal et al. 1974; Hood et al. 1982; Hood and Sonett 1982). Laboratory conductivity-temperature measurements were used to translate the lunar conductivity profile to a selenotherm that is roughly equivalent with the background, global heat flow derived by Wieczorek et al. (2006). The outer $500 \mathrm{~km}$ was poorly constrained due to the limited magnetometer bandwidth (higher frequencies are needed to resolve shallower depths). 
Fig. 2 The ARTEMIS spacecraft probe the lunar interior individually by the magnetotelluric method and jointly using the magnetic transfer function method. The investigation will provide constraints on core size, on the composition of the lower mantle, and on the nature of upper-mantle heterogeneity, particularly the anomalous Procellarum KREEP Terrane

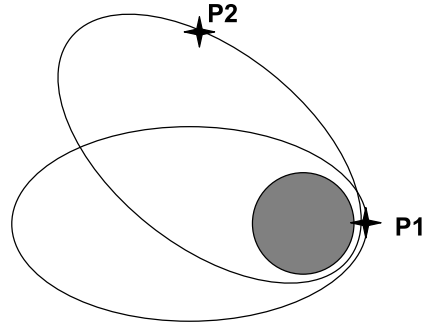

Magnetotail Primary Field

Transfer-Function Sounding: Measure B on P1, P2 Magnetotelluric Sounding: Measure E and B on P1

In spite of these advances, significant questions remain. The International Lunar Network Science Definition Team Report (1998) identified three new investigations for electromagnetic sounding: (1) determine the structure of the outer $500 \mathrm{~km}$ (upper mantle and crust) that may contain key information on early lunar evolution, specifically, the extent of the magma ocean and the origin of major provinces; (2) provide tighter bounds on the conductivity of the lower mantle (500-1400 km depth), that may constrain trace amounts of $\mathrm{H}_{2} \mathrm{O}$ (Saal et al. 2008; Grimm and McSween 2009); and (3) determine whether the Moon has a core, and is it metal or molten silicate.

The fundamental quantity that must be measured in any induction experiment is the frequency dependent electromagnetic impedance $Z$. The most important constraint is that two known quantities are always necessary (Grimm 2009), e.g., Ohm's Law $Z=V / I$. Apolloera lunar studies used the distantly-orbiting Explorer 35 to determine the source magnetic field, and compared this to Apollo 12 surface measurements that represent the sum of source and induced fields. This is the Transfer-Function technique (see Fig. 2). Alternatively the Magnetotelluric Method can provide a complete sounding from individual platforms using orthogonal horizontal components of the electric and magnetic fields (e.g., Vozoff 1991; Simpson and Bahr 2005; Grimm and Delory 2008). The plane-wave impedances determined by the Magnetotelluric Method are extendable to spherical geometry when skin depths become comparable to the planetary radius (Weidelt 1972; see also Schubert and Schwartz 1972).

With these considerations, we have defined an electromagnetic sounding investigation for ARTEMIS. Transfer Function soundings can be performed when the Moon is in the Earth's magnetotail, both probes are in daylight, one probe is near periapsis $(\sim 100 \mathrm{~km})$, and the other at least several lunar radii away (including up to apoapsis $\sim 19,000 \mathrm{~km}$ ). This ensures that the distant probe measures only the primary field while the periapsis probe measures the sum of the primary and secondary fields. Only the fluxgate magnetometers are necessary. This geometry contrasts with the free-solar-wind environment used for Apollo Transfer Function soundings. Here, the dayside confining plasma layer would have required the second measurement (primary+secondary field) to be made at or near the surface, well below the ARTEMIS periapsis altitude. Measurements with both spacecraft in the wake are also possible, although it may be more difficult to separate the relevant signals in the hot tenuous plasma in this shadowed region, where spacecraft charging may reach extremes.

The probe periapses of $100 \mathrm{~km}$ restrict the minimum investigation depth to a few hundred km or greater. They may glimpse some upper mantle structure but will certainly probe the lower mantle, especially given that measurements may be made during some tens of minutes around periapsis. Close study of field-line deflection over many periapses passages may be used to detect the core (Russell et al. 1982; Hood et al. 1999). 
ARTEMIS will conduct the first extraterrestrial tests of the Magnetotelluric Method, using both electric (EFI) and magnetic (FGM) records during periapsis passages. While these measurements may be possible under a variety of conditions and geometries, they are likely best conducted in sunlight while in the magnetotail. As these are the same conditions favoring Transfer Function soundings, results should be identical: discrepancies will highlight the kinds of data processing and/or auxiliary information needed to extract the electromagnetic part of the electric-field.

With its 1.5-yr mission at low periapses, ARTEMIS will have both data redundancy (signal integration) and geographic coverage around the equator. The ARTEMIS mission will advance our understanding of the deep lunar interior and provide a baseline for nextgeneration lunar and planetary electromagnetic sounding.

\subsection{Magnetic Anomaly Interactions}

Maps for lunar crustal magnetic fields remain significantly undersampled and the causes of lunar crustal magnetization remain uncertain. Local enhancements in the crustal magnetic field can shield the lunar surface from space weathering caused by ion sputtering and generate mini-magnetospheres whose effects can be felt far from the lunar surface. ARTEMIS will provide the combination of in situ and remote observations needed to substantially improve our global picture of crustal magnetism and then determine its effects on its immediate environment.

\subsubsection{Causes of Crustal Anomalies}

Patterns of crustal magnetization preserve a record of planetary evolution. Thermoremanent magnetization in the presence of a dynamo magnetic field is the dominant process by which the terrestrial crust has been magnetized, as is likely the case for Mars (Connerney et al. 1999). Alternating 'stripes' of magnetized seafloor crust at mid-ocean ridges were crucial in confirming geodynamo polarity reversals and the plate tectonics hypothesis (Vine and Matthews 1963; Vine and Wilson 1965). The origin(s) of lunar magnetism are far less certain: lunar sample measurements indicate the possible existence of a lunar dynamo from 3.6-3.9 billion years ago (Cisowski et al. 1983) with an order of magnitude decrease before and after that period.

There are many hypotheses for the source of the ambient field and the magnetization process. Steady magnetizing fields of both external (solar or terrestrial) and internal (lunar dynamo) origin have been proposed, as have transient fields generated by impacts (for reviews, see Fuller 1974, and Wieczorek et al. 2006). Thermoremanent magnetization is likely the dominant process for igneous lunar samples; however, shock remanent magnetization may be significant in lightly metamorphosed breccias, which carry the strongest and most stable remanent magnetization of all lunar samples because they contain more metallic iron grains (likely produced by meteoritic impacts) (Fuller et al. 1974; Fuller and Cisowski 1987). Mare basalts, on the other hand, contain less nanophase iron and generally have weaker remanent magnetization (Coleman et al. 1972).

With samples from only a handful of lunar landing sites, orbital magnetic measurements have been relied upon to study the global pattern of crustal magnetism. Two types of data have been used: magnetometer and electron reflection measurements. Magnetometer data provide vector information but are limited by spacecraft altitude and the fact that lunar crustal magnetic fields at satellite altitudes are typically very weak: $\sim 2$ and $\sim 3$ orders of magnitude weaker than the terrestrial and Martian cases respectively (Connerney 
et al. 2004). Electron reflection measurements invoke magnetic mirroring and use magnetometer observations together with electron energy and angular distributions measured from orbit to estimate the magnetic field magnitude at the lunar surface (Anderson et al. 1975; Lin et al. 1976). Electron reflection data have the virtue of being particularly sensitive to the weakest crustal fields (Halekas 2003; Mitchell et al. 2008).

Electron reflection and magnetometer measurements from the Apollo 15 and 16 subsatellites (Hood et al. 1981; Lin et al. 1988) and the Lunar Prospector spacecraft (Hood et al. 2001; Halekas et al. 2001; Mitchell et al. 2008) have enabled global mapping of lunar crustal fields. However, the map is severely undersampled, with significant noise and pixelization clearly visible (see Fig. 3), accounting for much uncertainty in geophysical interpretation. ARTEMIS ESA, with better energy resolution than Lunar Prospector or Apollo, will, along with ARTEMIS magnetometer measurements, enable more accurate correction for the effects of electrostatic potentials between the lunar surface and the spacecraft (Halekas et al. 2002b; Mitchell et al. 2008) and therefore provide more accurate crustal field estimates. The two ARTEMIS probes will make many hundreds of low altitude $(<400 \mathrm{~km})$ electron reflection measurements within $10^{\circ}\left(20^{\circ}\right.$ if optimized) of the equator on every periselene pass, resulting in better spatial coverage and higher fidelity crustal field maps in these regions than currently exist.

Existing maps show weaker fields over the mare basalts compared with the highlands (in agreement with the aforementioned sample studies) and reveal that the largest area of strong crustal fields lie in regions diametrically opposed to the Imbrium, Serenitatis, Crisium and Orientale impact basins (see Fig. 3). This led to the hypothesis that crustal magnetization is associated with basin-forming impacts. According to this hypothesis, the hypervelocity ( $>10 \mathrm{~km} / \mathrm{s}$ ) impacts that form such large basins produce a plasma cloud that expands around the Moon, compressing and amplifying any pre-existing ambient magnetic field at the antipodal point (Hood and Huang 1991), where the focusing of seismic energy and impact of basin ejecta may result in substantial shock remanent magnetization (Hood and Artemieva 2008). A key element of this hypothesis is that it does not require a global dynamo field, although recently Wieczorek and Weiss (2010) have shown that the simplest explanation for some of the strongest anomalies like Reiner-Gamma involves a steady, dynamo magnetic field. Better maps enabled by ARTEMIS in the equatorial regions will help to elucidate the mechanism of antipodal magnetization by allowing us to better constrain the characteristics of the remanent crustal remanent magnetization.

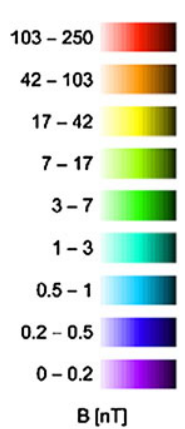

Fig. 3 Impact basin rims (white circles, for Imbrium the interior ring is also shown) and their antipodes (black circles), superimposed on a map of total surface magnetic field strength averaged over $5 \times 5^{\circ}$ bins (Mitchell et al. 2008) 
In summary, the ARTEMIS electron reflection data set will substantially augment existing observations, enabling greater resolution in the equatorial regions and thus more confident interpretation. In so doing, ARTEMIS will improve our knowledge of the interconnected histories of lunar impacts, basin formation, magmatism and the ancient lunar dynamo.

\subsubsection{Effects of Crustal Anomalies on the Plasma Environment}

The sub-ion inertial scale lengths associated with crustal magnetic anomalies raise a host of questions about the nature of their interaction with the solar wind. Does the solar windanomaly interaction produce a shock? Do the magnetic anomalies shield a region from the solar wind, generating a lunar "mini-magnetosphere" that stands off and deflects the solar wind (Hood and Schubert 1980; Hood and Williams 1989; Hood 1992; Lin et al. 1998; Harnett and Winglee 2000)? Past and current missions have led to great progress on these questions, but ARTEMIS stands ready to add to our knowledge, thanks to its comprehensive plasma instrumentation and two-probe design. ARTEMIS offers the unprecedented (at the Moon) capability to measure full 3-D plasma distributions and electric fields, allowing better understanding of the microphysics of the interaction, and to measure the properties of the plasma just upstream from the anomaly region, allowing separation of spatial and temporal effects. The results may be applicable to other bodies, including Mars (e.g., Breus et al. 2005), asteroids such as Gaspra and Ida (Kivelson et al. 1995), and possibly Vesta (Vernazza et al. 2006).

Explorer 35 and the Apollo subsatellites first observed the effects of magnetic anomaly interactions near the limb in the form of compressional waves propagating downstream. Lunar Prospector, however, observed the effects extending to $\sim 100 \mathrm{~km}$ altitudes, sometimes well upstream $\left(>45^{\circ}\right)$ from the limb and their apparent sources, seemingly requiring the formation of a shock (Lin et al. 1998). The coincident enhancements in the upstream electron density, electron flux, magnetic field strength, and whistler mode wave activity upstream seen in Fig. 4 all suggest the presence of a shock (Lin et al. 1998; Halekas et al. 2006b, 2007, 2008b). By contrast, simulations for the solar wind interaction with dipoles with dimensions comparable to lunar anomalies only predict whistler or magnetosonic wakes, not shocks (Omidi et al. 2002). Some simulations suggest that the non-dipolar nature of lunar magnetic sources might increase the efficiency of the interaction (Harnett and Winglee 2003), perhaps resolving this discrepancy. Also, surface influences, including secondary electrons, photoelectrons, and dust, may turn out to play a role. To determine whether lunar anomalies do in fact form shocks (Halekas et al. 2006a, 2006b), we need the capability of ARTEMIS plasma instrumentation, which thanks to the spinning spacecraft and spacecraft potential measurements, can make precise measurements of bulk properties and anisotropies of the 3-D velocity distributions of both electrons and ions.

\subsubsection{Space Weathering: Effects of Crustal Anomalies on the Lunar Surface}

The Moon, like any other body exposed to the harsh space environment, is subject to galactic and solar cosmic rays, irradiation, implantation, and sputtering from solar wind particles, and bombardment by meteorites and micrometeorites. This exposure causes radiation damage, chemical changes, optical changes, erosional sputtering, and heating, all essential parts of the process called space weathering. Space weathering is important because these processes affect the physical and optical properties of the surface of many planetary bodies. To properly interpret remote sensing observations, it is critical to understand the effects of space weathering. 

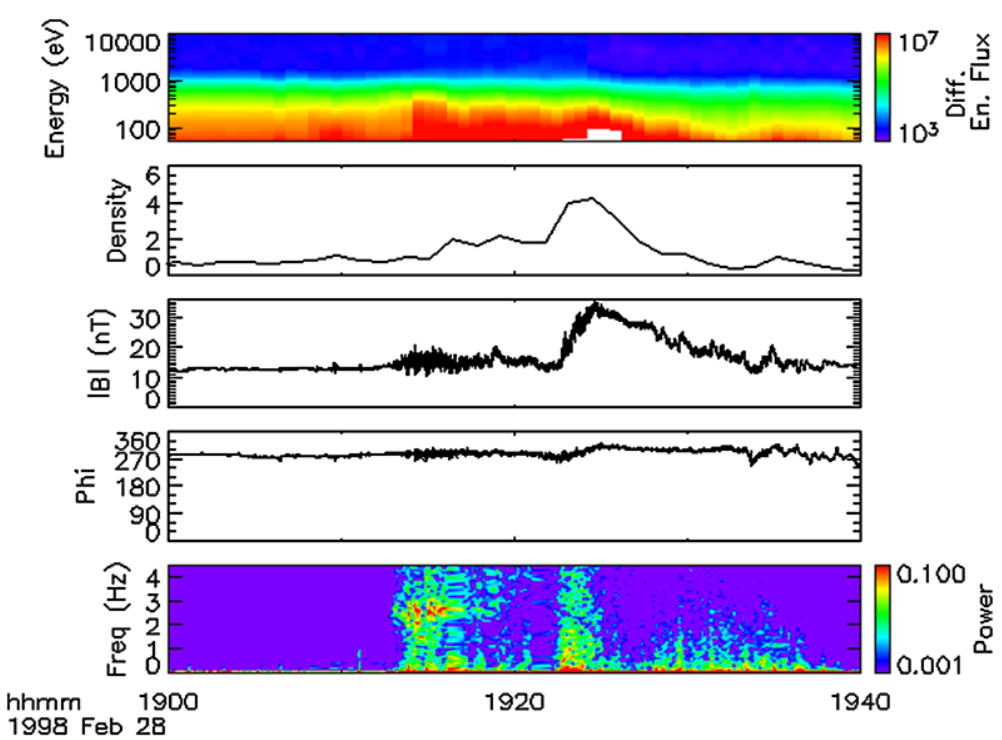

Fig. 4 Lunar Prospector electron energy spectra, electron density, and magnetic field strength, longitude, and power frequency spectra suggestive of a shock

A number of observations suggest that magnetic anomalies can shield the surface from the solar wind, with clear implications for surface weathering, and possible implications for the formation of the lunar albedo markings often observed near strong crustal magnetic sources (e.g., Hood and Schubert 1980; Richmond et al. 2005). Lunar Prospector observations of low altitude density cavities above a strong crustal anomaly lend some support to this idea (Halekas et al. 2008a). More recent Kaguya observations of ion flow deceleration, electron heating, and no scattered protons above strong crustal anomalies also suggest surface shielding (Saito et al. 2010). Finally, Chandrayaan has provided observations indicating no scattered neutral atoms from the same region discussed by Halekas et al. (2008a), strongly suggesting the existence of a magnetically shielded surface region (Wieser et al. 2010).

The two ARTEMIS probes will enable more observations like those by Kaguya, but with more comprehensive plasma instrumentation, and the ability to compare observations upstream and outside of the anomaly region with those inside. This will enable separation of temporal and spatial effects, and allow researchers to clearly determine how the anomaly interaction affects the incoming solar wind flow, including whether and how electrons and ions de-couple near anomalies, what mechanisms produce electric fields, and the nature and distribution of particle heating and wave-particle interactions.

\section{ARTEMIS Heliophysics Science Objectives}

ARTEMIS will address the host of heliospheric science objectives illustrated in Fig. 5. Topics include reconnection, particle acceleration, and turbulence in the magnetotail and interplanetary space, and the structure and evolution of the Moon's plasma wake. This section presents a selection of topics to be considered as a function of location in orbit: addressing successively the Earth's magnetotail, the solar wind, and the lunar wake. 


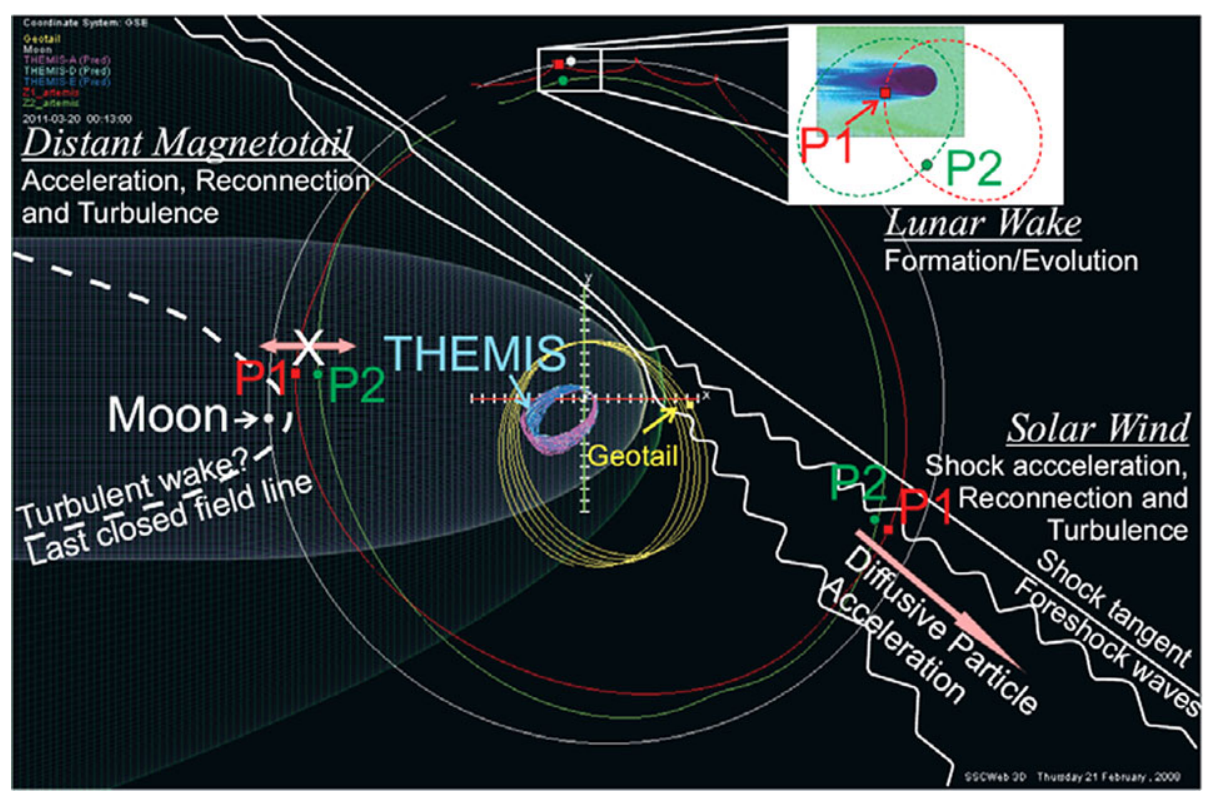

Fig. 5 ARTEMIS heliophysics scientific objectives address topics including reconnection, particle acceleration, and turbulence in the solar wind and Earth's magnetotail and the formation and evolution of the lunar wake

\subsection{In the Magnetotail}

The Earth's magnetotail comprises two lobes with oppositely directed magnetic fields divided by an equatorial current sheet embedded within a plasma sheet marked by enhanced densities and temperatures but depressed magnetic field strengths. Conditions within the near-Earth magnetotail are relatively well-understood, thanks in large measure to the multipoint measurements returned by the THEMIS mission. By contrast, fleeting glimpses of the distant magnetotail by the Explorer-33 and -35, ISEE-3, Geotail, and Wind missions have raised numerous questions that can only be answered by examining extensive multipoint in situ observations. Potential research topics include the structure of the distant magnetotail during prolonged intervals of northward IMF orientation, the length, shape, and occurrence patterns for reconnection lines at lunar distances, the evolution of the heated and accelerated flows ejected by these reconnection lines, the shape, dimensions, internal structure, and evolution of antisunward-moving plasmoids and flux ropes, boundary waves on the surface of the magnetotail, and the nature of turbulence within the Earth's magnetotail plasma sheet.

ARTEMIS will provide the observations needed to address these questions. Together with the Moon, the two ARTEMIS spacecraft will spend about 4.5 days of each 28-day orbit about the Earth within the magnetotail at lunar distances, and 20 to 30 hours per orbit within the plasma sheet (Hapgood et al. 2007). With interspacecraft separation distances ranging from $500 \mathrm{~km}$ to $20 R_{\mathrm{E}}$, the two spacecraft will be well situated to determine not only the steady-state macrostructure of the distant magnetotail, but also the meso- and micro-scale characteristics of the superimposed transient events as a function of solar wind conditions. 


\subsubsection{Structure of the Magnetotail}

The first question to be addressed concerns the structure of the distant magnetotail during prolonged intervals of northward IMF. Magnetosheath momentum imparted to the magnetosphere drags magnetospheric magnetic field lines antisunward to form the Earth's magnetotail. During periods of southward IMF orientation, this momentum is imparted via the reconnection of magnetospheric and interplanetary magnetic field lines on the dayside magnetopause. Theory predicts, and observations confirm, that the Earth's polar caps lie on open magnetic field lines leading to interplanetary space during intervals of southward IMF orientation (Dungey 1961). The situation during periods of northward IMF (NBZ) remains unclear. Reconnection on the dayside equatorial magnetopause ceases, terminating the production of open magnetic field lines. Reconnection within the magnetotail continues, transforming open lobe into closed plasma sheet magnetic field lines. The likelihood of dual lobe reconnection, which also closes open lobe field lines while appending magnetosheath magnetic field lines to the magnetosphere, increases. The magnetotail may become topologically closed during prolonged NBZ periods.

Observations from low altitude polar orbiting spacecraft like DMSP can be used to infer the polar cap size, the amount of open flux in the lobes, and whether or not the magnetotail ever closes. Low-altitude observations indicate that the size of the open magnetic field line region within the polar cap slowly diminishes during periods of strong and persistent northward IMF (Newell et al. 1997). However, spacecraft may fail to enter the polar caps when their size diminishes, resulting in a mistaken interpretation that they are absent. Furthermore, their identification can become more difficult during extended NBZ periods because the characteristics of the precipitating particles used to identify their boundaries may change. In the absence of clear predictions for the shape and geometry of the magnetotail and its plasma and magnetic field characteristics during NBZ periods, in situ observations of the magnetotail have also generally been inconclusive. A chance Wind encounter with a strongly deformed and twisted magnetotail some $125 R_{\mathrm{E}}$ from Earth during an extended NBZ period on October 22-24, 2003 indicates an open magnetotail; albeit one with highly unusual plasma and magnetic field properties (Øieroset et al. 2008).

Global numerical models for the magnetosphere predict strikingly different magnetospheric topologies during NBZ periods. Many predict magnetospheres that close near Earth, as envisioned by Axford and Hines (1961). Others predict closed magnetospheres that extend much further, perhaps in response to viscous momentum transfer enhanced by the Kelvin-Helmbholtz instability (Miura 1984). Predicted lengths for the closed magnetotails vary from as little as $\sim 30$ to several hundred $R_{\mathrm{E}}$ (Usadi et al. 1993; Watanabe and Sato 1990; Ogino et al. 1992; Fedder and Lyon 1995; Gombosi et al. 1998; Guzdar et al. 2001). However, not all models predict tail closure during NBZ periods. Raeder et al. (1995) presented idealized simulations of a NBZ period where the tail stayed open for several hours. In fact, Raeder (1999) argued that the prediction of a closed tail in most numerical MHD models was due to excessive numerical diffusion, a point discussed in further detail by Gombosi et al. (2000) and Raeder (2000).

Figures 6 and 7 present Open Geospace General Circulation Model (OpenGGCM) predictions for the size and shape of the Earth's magnetotail during the October 22-24, 2003 Wind event. The width of the magnetotail diminishes with distance downstream, but an open magnetotail that flaps dawnward and duskward is still present at lunar distances. Torques applied by the IMF twist the magnetotail, causing the southern lobe to shift northward at dusk and the northern lobe to shift southward at dawn (e.g., Sibeck et al. 1985)

ARTEMIS will provide the observations needed to determine the size, shape, and internal structure of the Earth's magnetotail as a function of independently measured solar 


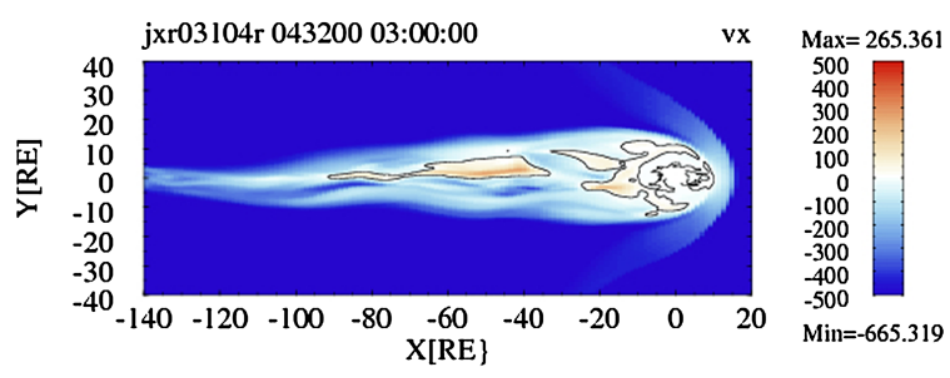

Fig. 6 OpenGGCM simulation predictions for the component of the plasma velocity along the Sun-Earth line during the October 22-24, 2003 strong NBZ event. Magnetotail widths diminish steadily with distance antisunward
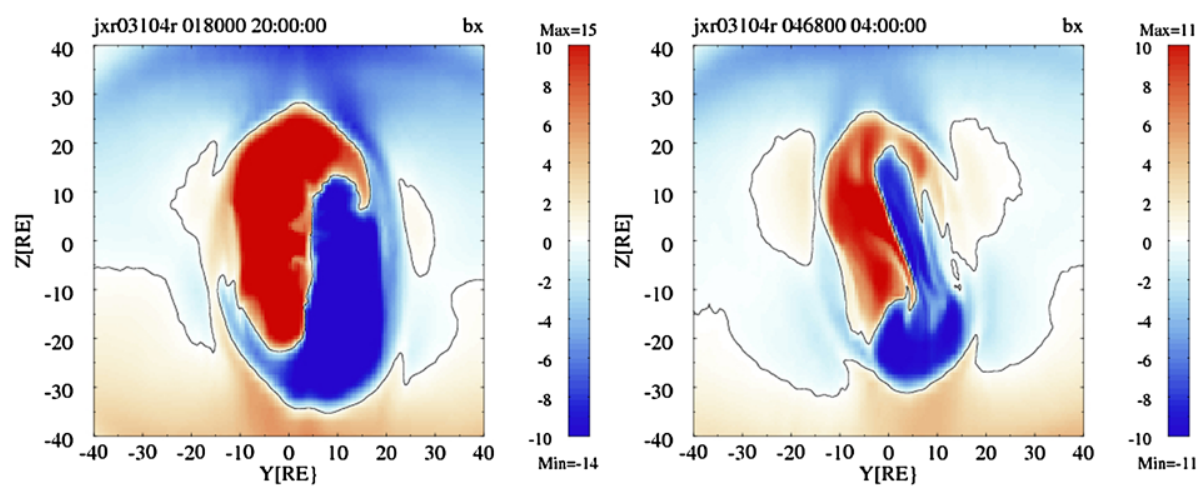

Fig. 7 OpenGGCM predictions for sunward (red) and antisunward (blue) magnetic field polarities within magnetotail cross-sections $60 R_{\mathrm{E}}$ from Earth at 20:00 UT on October 22, 2003 and 04:00 UT on October 23, 2003. The magnetotail is severely twisted and deformed

wind and geomagnetic conditions, during both northward and southward IMF orientation. Two point ARTEMIS measurements will aid in determining the orientations of the various boundaries and the characteristics of their flapping motions. One of the two ARTEMIS probes will frequently monitor magnetosheath conditions immediately outside the distant magnetotail magnetopause while the other measures (e.g.) electron pitch angle distributions at the magnetopause and deeper within the magnetotail, the key information needed to reveal magnetic field line topology and history in those regions (Hasegawa et al. 2005; Øieroset et al. 2008). Multi-season and multi-year observations will permit researchers to test whether dual lobe reconnection for northward IMF orientations becomes more efficient when the Earth's dipolar tilt diminishes (Li et al. 2008).

\subsubsection{Plasmoids and Flux Ropes: Reconnection Within the Near-Earth Magnetotail}

As illustrated in Fig. 8, bursts of reconnection along extended lines in the near-Earth magnetotail release immense antisunward-moving flux ropes, or plasmoids, that carry vast quantities of plasma and magnetic flux antisunward through the Earth's plasma sheet. Spacecraft in the vicinity of the plasma sheet may directly enter the plasmoids, while spacecraft within the magnetotail lobes record perturbation signatures known as traveling compression regions (TCRs). In conjunction with Geotail, Cluster, and baseline THEMIS observations of 


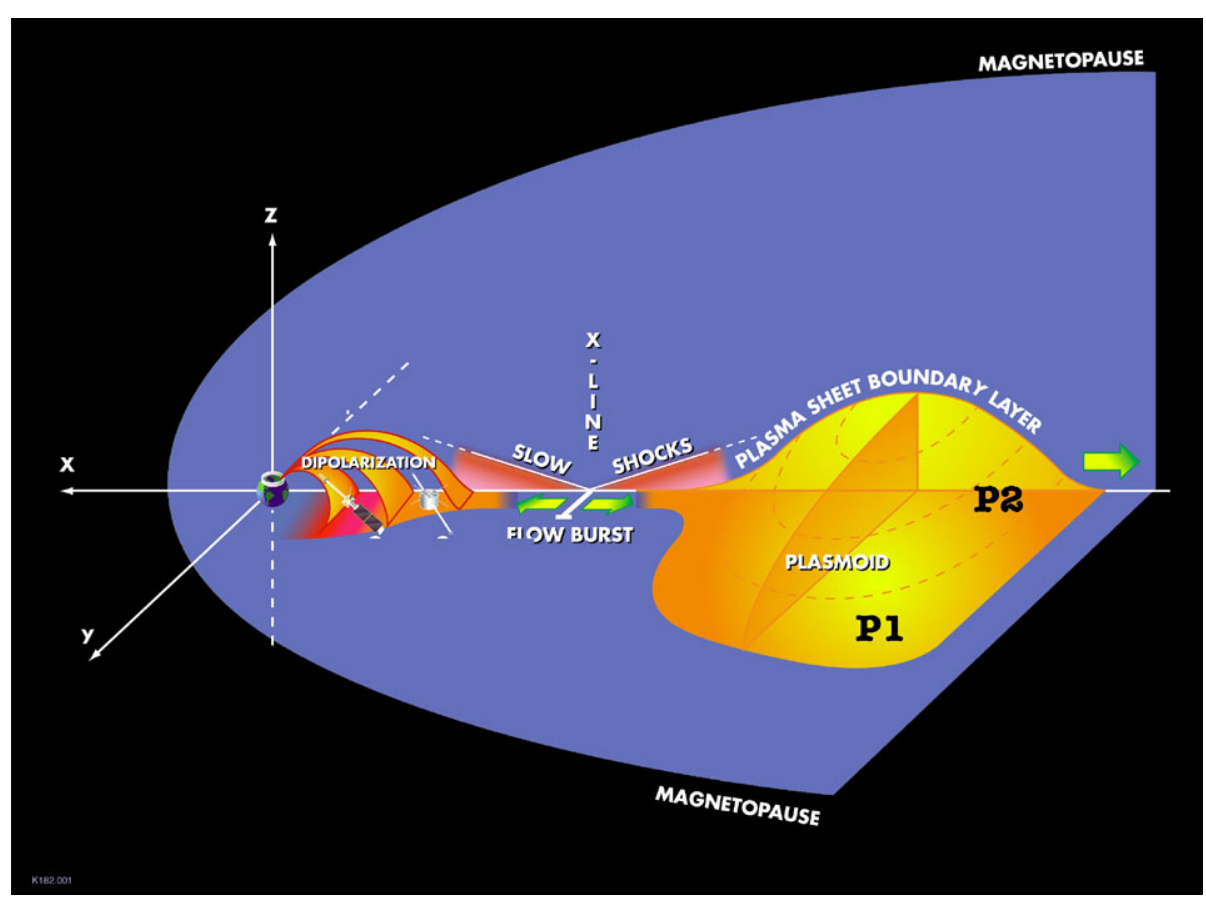

Fig. 8 Reconnection at an $x$-line in the near-Earth magnetotail results in dipolarizations of the magnetospheric magnetic field and releases enormous plasmoids that move antisunward through the magnetotail. When separated transverse to the axis of the magnetotail, ARTEMIS probes P1 and P2 will be well-situated to determine the dimensions, shape, and internal structure of the plasmoids

the near-Earth magnetotail, and employing its own observations separated by $1-10 R_{\mathrm{E}}$ along the Sun-Earth line, ARTEMIS will provide the observations needed to determine the internal structure of plasmoids and track their evolution down the magnetotail.

Plasmoid dimensions are a crucial factor in determining their significance to the solar wind-magnetosphere interaction. Plasmoid diameters increase by a factor of 2-3 from neartail to lunar distances (Ieda et al. 1998), but the reasons for this growth remain unclear. Part of the growth results from expansion in response to lower ambient pressures in the distant magnetotail, part may be due to continued reconnection at reconnection lines moving down the magnetotail with the plasmoids, and part may be due to the coalescence of smaller plasmoids to form larger plasmoids as they are transported tailward. The cross-tail extent of plasmoids remains unknown. Although plasmoids in the distant magnetotail often exhibit force-free structures, their magnetic topology remains uncertain. Magnetic field lines within plasmoids may be closed loops, connected to Earth, or connected to the interplanetary medium.

Azimuthal probe separations of $1-10 R_{\mathrm{E}}$ will enable ARTEMIS to determine the crosstail extent, orientation, and shape (using minimum variance analyses of the magnetic field) of plasmoids. Radial separations will provide the observations needed to determine how rapidly plasmoid diameters change with distance downstream (e.g., Slavin et al. 1999). Grad-Shafranov reconstruction techniques (Hasegawa et al. 2004) will be used to determine the plasma and magnetic field structure within the plasmoids from time series measurements and an assumption that they are in near pressure balance with their immediate environment. 
Fig. 9 Reconnection along an extended and curved line in the distant magnetotail. Because the line demarcates the boundary between sunward (Earthward) and antisunward flows, its location can be determined when ARTEMIS probes $\mathrm{P} 1$ and $\mathrm{P} 2$ are radially separated. By contrast, its extent can be determined when the two spacecraft lie separated transverse to the axis of the magnetotail

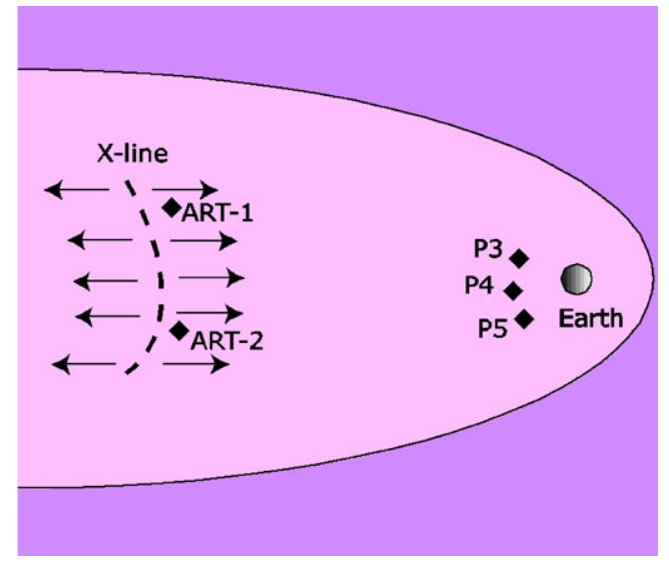

High time resolution ARTEMIS measurements of multiple pitch angle distributions during events with anticipated time durations ranging from 100 to $200 \mathrm{~s}$ will not only offer an opportunity to determine their magnetic field topology, but also to test predictions concerning reconnection-related electron energization in contracting magnetic islands (Drake et al. 2006).

\subsubsection{Occurrence Patterns for Reconnection in the Distant Magnetotail}

By contrast to the situation in the near-Earth plasma sheet, where reconnection associated with geomagnetic substorms is often bursty and patchy (Baumjohann et al. 1989, 1990; Angelopoulos et al. 1992), ISEE-3, Geotail, and Wind observations of long-duration reconnection-heated plasma jets suggest that reconnection in the distant magnetotail plasma sheet is often quasi-steady and large-scale. As illustrated in Fig. 9, reconnection may occur along curved reconnection lines whose cross-tail extent depends upon the IMF orientation (Feldman et al. 1985; Nishida et al. 1995; Angelopoulos et al. 1996; Øieroset et al. 2000). In the absence of multi-point measurements, we do not know either the conditions favoring localized and extended reconnection in the distant magnetotail or the shape of the reconnection line. Two-point ARTEMIS observations enable both the occurrence patterns of reconnection and its extent across the lunar magnetotail to be determined as a function of guide field strength, geomagnetic, and solar wind conditions.

\subsubsection{Heating, Particle Acceleration, and Plasma Transport at Distant Reconnection Lines}

The plasma sheet within the distant magnetotail is an excellent location to study magnetic reconnection. By contrast to the near-Earth magnetotail, there is no heating in response to flow braking against the dipolar magnetic field lines. Furthermore, the finite mantle densities in the distant magnetotail mean that plasma parameters in the inflow region can be better characterized than they would be in the very tenuous near-Earth magnetotail. Topics for investigation by ARTEMIS include the process(es) by which reconnection heats plasmas, particle acceleration in reconnection geometries, and the subsequent motion of the reconnected plasma.

The degree to which reconnection heats ions, and the factors controlling this heating, are presently not known. Results from some recent simulations suggest that the heating is proportional to the speed of the outflowing Alfvénic jets, which are in turn related to the 
Alfvénic velocities of the inflowing plasma (Drake et al. 2009). The two ARTEMIS spacecraft will provide numerous opportunities to simultaneously compare inflow and outflow parameters.

Particles can be accelerated to suprathermal energies by drifting along X-lines, but also by Fermi-acceleration in the collapsing bubbles that surround O-lines (Hoshino et al. 2001; Drake et al. 2006). Wind observations of a single event in the distant magnetotail (at $X_{\mathrm{GSE}}=-60 R_{\mathrm{E}}$ ) provide evidence for electron energization to at least $300 \mathrm{keV}$ (Øieroset et al. 2002). Two-spacecraft ARTEMIS observations of plasma flows and magnetic field components normal to the current sheet are essential to discriminate between (and track the motion of) X-lines and O-lines. ARTEMIS will provide the previously unavailable high resolution plasma and electric field measurements needed to determine the causes for electron energization.

Steady reconnection in the distant magnetotail should eject high-speed plasma flows and energized particles both Earthward and away from the Earth. The occurrence patterns and extents of distant magnetotail reconnection lines will be determined from two-point ARTEMIS observations of the Earthward (antisunward) streaming energetic particles in the plasma sheet boundary layer, sunward (antisunward) flows, and northward (southward) magnetic field components in the current sheet expected sunward (antisunward) from the reconnection line. There are indications that most of the sunward plasma jets from the distant tail do not reach the near-Earth plasma sheet (e.g., Øieroset et al. 2004). Working in conjunction with the three inner THEMIS spacecraft, Cluster, and Geotail, ARTEMIS will establish the relationships between plasma flows in the lunar and near-Earth plasma sheet to determine the fate of reconnection jets generated in the distant tail. The results will help distinguish between models in which the flows are decelerated and/or deflected towards the flanks.

\subsubsection{Boundary Waves, Flux Transfer Events, and Turbulence in the Magnetotail}

The Kelvin-Helmholtz (KH) instability may play an important role in transferring solar wind mass, momentum, and energy into the Earth's magnetotail (Fujimoto and Terasawa 1994; Miura 1984, 1992), perhaps dominating the overall solar wind-magnetosphere interaction during intervals of northward IMF orientation when reconnection shuts down on the dayside magnetopause. The instability is most likely along the low-latitude flank magnetopause, where and when flow shears lie nearly perpendicular to weak northward magnetosheath and magnetospheric magnetic fields (Southwood 1968). As illustrated in Fig. 10, the amplitudes and wavelengths of waves generated by the Kelvin-Helmholtz instability should grow

Fig. 10 Illustrating the expected evolution of the Kelvin-Helmholtz instability from a linear stage on the dayside magnetopause to a nonlinear stage on the flanks of the distant magnetotail

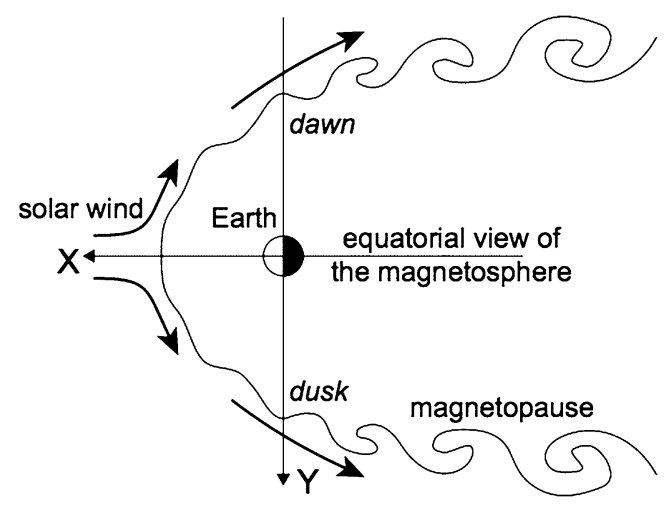


with downstream distance. The waves may merge into larger vortices (Miura 1997), perhaps with steepened trailing edges (Chen and Kivelson 1993). They may also generate fast-mode shocklets (Lai and Lyu 2006) or induce turbulence (Matsumoto and Seki 2007). Simulations that include the effects of coupling with the ionosphere and/or the tail lobes indicate that finite plasma sheet thicknesses may suppress the nonlinear growth of the instability (Galinsky and Sonnerup 1994; Takagi et al. 2006).

Observations indicate that the $\mathrm{KH}$ instability is relatively common on the flanks of the near-Earth magnetotail, occurring for the predicted solar wind conditions and exhibiting the expected signatures (Fairfield et al. 2000; Hasegawa et al. 2006). The structural properties of the $\mathrm{KH}$ waves are similar on the dawn and dusk flanks of the near-Earth magnetosphere (Hasegawa et al. 2006; Nishino et al. 2011). However, non-MHD processes that depend on the polarity of the field-aligned vorticity could lead to dawn-dusk asymmetries further downstream (Nakamura et al. 2010).

Although the distant magnetotail magnetopause is constantly in motion, and this motion has occasionally been attributed to the KH instability (Sibeck et al. 1987), little is known about the properties of the instability at downstream distances beyond $30 R_{\mathrm{E}}$. ARTEMIS will provide the simultaneous two-point observations of magnetosheath and magnetosphere needed to test instability criteria and determine the velocities, amplitudes, and wavelengths of the resulting boundary waves. Both the two-point observations, and comparisons with simultaneous Cluster and Geotail traversals of the near-Earth magnetopause, will help determine whether waves breakup, coalesce, expand, stabilize, or simply propagate downstream. The same two point observations will be examined for evidence of KH-generated reconnection, diffusion, turbulence, shocklets, and dawn/dusk asymmetries (e.g. Chaston et al. 2007).

Observations on the dayside and near-Earth flank magnetopause reveal the presence of twisted ropes of interconnected magnetosheath and magnetospheric magnetic field lines marked by transient magnetic field strength increases and bipolar magnetic field signatures normal to the nominal magnetopause. Interpreted as flux transfer events or FTEs, the flux ropes provide evidence for the frequent occurrence of unsteady reconnection at the magnetopause (Russell and Elphic 1978; Raeder 2006). The significance of the events to the overall solar wind-magnetosphere interaction remains to be determined. If entirely disconnected from the ionosphere, the events may simply advect antisunward with the magnetosheath flow, maintaining their orientation and helical magnetic field geometry, and represent nothing more than an interesting curiosity. However, if one end of the flux ropes remains anchored in the ionosphere while the other extends into interplanetary space, the events may account for a significant transfer of solar wind mass, energy and momentum to the magnetosphere. In this case, the flux ropes will stretch out parallel to the Sun-Earth line and sink into the magnetotail lobes (Sibeck and Siscoe 1984). Two-point ARTEMIS measurements will help determine event motion, orientation, length, and topology at lunar distances, thereby providing crucial information concerning their role in the solar wind-magnetosphere interaction.

Turbulent dissipation is an effective mechanism for heating fluids and transferring mass, momentum and energy. Although turbulence is ubiquitous in space plasmas, much remains to be determined concerning its initiation and subsequent evolution. Turbulence in the nearEarth plasma sheet has been studied using Cluster (Weygand et al. 2007). The dissipation range was on the order of the ion inertial lengths or gyroradius ( $\sim$ few hundred $\mathrm{km})$ and the correlation coefficients diminished to zero beyond scales of $3 R_{\mathrm{E}}$. Flow fluctuations were small relative to the sound and Alfvén speeds (except during dynamic conditions). By contrast, flow fluctuations are comparable to the sound and Alfvén speeds in the distant 
magnetotail and therefore dynamically and energetically important. Theory and simulations point towards magnetotail reconnection and velocity shears at the flanks as likely drivers of plasma sheet turbulence. Both drivers can affect energy circulation and particle transport within the magnetosphere. Characterizing the nature of the fluctuations, and determining their origin and dissipation is therefore important for global circulation. It is quite likely that the distant tail also exhibits an inertial range of turbulence. ARTEMIS will use varying interspacecraft separation distances to characterize turbulence over a wide range of spatial scales.

ARTEMIS may also observe turbulence at the distant magnetotail magnetopause. During periods of northward or nearly radial IMF orientation, reconnection on the high-latitude and flank magnetopause results in high speed antisunward flows in a boundary layer of open magnetic field lines disconnected from Earth. High-latitude reconnection can continue for hours under northward IMF conditions (Frey et al. 2003; Hasegawa et al. 2008). The distant high-latitude magnetopause is an ideal region to study turbulence in reconnection outflows. By contrast to the dayside magnetopause, the effects of Alfvén waves reflected from the ionosphere need not be considered. And by contrast to the near-Earth plasma sheet, the resistance of the dipolar magnetic field region need not be addressed. ARTEMIS will determine whether or not a quasi-steady inertial range of turbulence is attained as a result of energy injection via reconnection. The two-point measurements permit estimations of the wavelength (instead of or in addition to the frequency) of the associated waves (Chaston et al. 2008). A spacecraft in the magnetosheath can provide information about the nature of magnetosheath turbulence and whether conditions favor high-latitude reconnection, thereby helping discriminate between the effects of magnetosheath and reconnection-induced turbulence.

\subsection{In the Solar Wind}

Together with the Moon, the ARTEMIS spacecraft will spend most of their orbit about the Earth in the solar wind. Here ARTEMIS provides a unique opportunity to address longstanding questions concerning the physics of the foreshock, interplanetary shocks, reconnection in the solar wind, and plasma turbulence. In particular, ARTEMIS will be used to:

- examine how the bow shock, and collisionless shocks in general, accelerate particles to high energies;

- study the structure of interplanetary shocks, and examine how non-planar structure can, for example, influence the production of type II radio emission;

- explore reconnection in the solar wind, and provide the high time resolution measurements required to understand low magnetic shear reconnection, which recent measurements suggest predominates in the heliosphere.

- fill in the gaps in our knowledge of solar wind turbulence by providing cross- observations of solar wind features from spacecraft separated by previously inaccessible interseparation distances of $11,000-50,000 \mathrm{~km}$.

\subsubsection{Shock Physics-Particle Acceleration in the Terrestrial Foreshock}

In addition to mediating the flow of super-magnetosonic plasma, collisionless shocks also act as sites for particle acceleration (see, e.g., reviews by Terasawa 2003; Burgess 2007). If the upstream magnetic field is not perpendicular to the shock normal, then a portion of the inflowing plasma can escape back into the upstream region rather than being processed by the shock; the interaction of this backstreaming component with the inflowing plasma 
leads to wave generation and particle acceleration (Eastwood et al. 2005). The way in which collisionless shocks produce energetic particles is a problem of extremely broad astrophysical importance. The Earth's bow shock/foreshock is one of the best laboratories we have for studying in-situ the basic physical processes that govern shock particle acceleration (Burgess 2007).

Diffusive shock acceleration (Axford et al. 1977; Bell 1978a, 1978b; Blandford and Ostriker 1978) is widely cited as the process by which ions are accelerated to high energies at shocks. At the Earth's bow shock, ion energies extend to at least several MeV (Lin et al. 1974; Desai et al. 2000). Diffusive shock acceleration theories predict that the density of energetic ions falls exponentially with distance from the shock front into the upstream region. On a statistical basis, single spacecraft observations just upstream of the bow shock have shown that the energetic ion flux e-folding distance varies from $3.2+/-0.2 \mathrm{Re}$ at $10 \mathrm{keV}$ to $9.3+/-1.0 \mathrm{Re}$ at $67 \mathrm{keV}$ (Trattner et al. 1994). In a two-spacecraft case study using Cluster, Kis et al. (2004) and Kronberg et al. (2009) found the e-folding distance varied from $0.5 \mathrm{Re}$ at 11 to $2.8 \mathrm{Re}$ at $27 \mathrm{keV}$, and increased almost linearly with energy up to $\sim 120 \mathrm{keV}$. Although two-point observations provide a far more reliable measurement of the e-folding distance, the Kis et al. (2004) and Kronberg et al. (2009) multipoint analysis studied only one event, thus limiting their conclusions to high Mach number solar wind in a limited location (a few $R_{\mathrm{E}}$ upstream of the shock) over a limited time interval (8 hours of data due to the orbit of the spacecraft). Evidently a multi-point statistical survey is desirable and necessary to better understand the nature of accelerated particles upstream of the bow shock.

ARTEMIS will make extensive observations of the upstream region where particles are accelerated to high energies. ARTEMIS will not cross the sub-solar bow shock except during extreme solar wind conditions, e.g. very low solar wind Mach numbers (Farris and Russell 1994), that would in itself result in serendipitous scientific discoveries. The presence of two spacecraft allows correlation lengths parallel and perpendicular to the field to be studied in the key range of 0.1-20 $R_{\mathrm{E}}$ without the confounding effects of upstream variability-indeed observations can be quantified according to upstream conditions. Foreshock energetic particles will be observed both intermittently and continuously over many days, providing excellent statistics. The ARTEMIS probes will sample a much wider range of distances from the shock than previously accessible, at various distances from the tangent line and over a wide range of depths in the foreshock.

ARTEMIS will also provide experimental data that challenges the common use of linear diffusive shock acceleration theory. At the bow shock there is already incontrovertible evidence that the linear theory is insufficient; for example, magnetic field fluctuations do not exhibit the power law spectrum assumed by models over the required frequency range (Terasawa 1995). Although linear diffusive shock acceleration theory has been extended into the quasi-linear regime (where the energy flows from the particles to the waves, but the waves themselves are given by linear theory) (Lee 1983), experimental tests of quasi-linear theories at the Earth's bow shock have not been carried out (Burgess 2007). Furthermore, recent large-scale hybrid simulations predict that rather than continuing to diminish, the energetic particle flux approaches a constant at some point far upstream of the shock (Giacalone 2004). ARTEMIS measurements will help to establish the homogeneity of the upstream wave field, of key importance for developing more complex theories of particle acceleration in the foreshock.

Finally, as noted by Burgess et al. (2005), diffusive shock acceleration has also often been challenged by the hypothesis that all upstream ion enhancements at Earth are exclusively magnetospheric in origin (e.g. Sarris et al. 1987). Although studies have concluded that most upstream energetic ions do not originate in the magnetosphere (Gosling et al. 
1989), the presence of energetic magnetospheric oxygen in the foreshock has been reported (Mobius et al. 1986). The combination of ARTEMIS, making measurements in the foreshock, together with the remaining three THEMIS spacecraft at the magnetopause and in the magnetosphere measuring changes in energetic ions and the state of the magnetosphere, will allow the magnetospheric input of energetic particles to the shock to be deconvolved from diffusive shock acceleration better than ever before for a variety of solar wind conditions.

\subsubsection{The Structure of Interplanetary Shocks}

Interplanetary (IP) shocks energize particles, and observations in their vicinity can be used to test theories of particle acceleration. They are thought to be responsible for the production of Solar Energetic Particles (SEPs) in so-called gradual events. In one study of particular note, Kennel et al. (1986) used ISEE-3 observations of an IP shock to test the Lee (1983) quasilinear theory of diffusive shock acceleration. A number of disagreements were observed which could be explained by variations in the upstream conditions, but since data from only a single spacecraft were available, this could not be resolved. By simultaneously observing the conditions both up- and downstream of interplanetary shocks, and then generating statistics based on observations of many IP shocks during the course of the mission, ARTEMIS will provide new information about how IP shocks generate energetic particles. The routine acquisition of spin resolution ( $3 \mathrm{~s}$ ) plasma moments will be of particular importance in this regard. Figure 11 shows an example of an IP shock observed by both ARTEMIS spacecraft on 5 April 2010. At this time the spacecraft were in transition from terrestrial to lunar orbit.

Related to this issue is the nature of type II radio emission. Type II radio emissions are thought to be generated patchily in the region upstream from Coronal Mass Ejection (CME)driven interplanetary shocks (Bale et al. 1999; Pulupa and Bale 2008), and are a useful tool

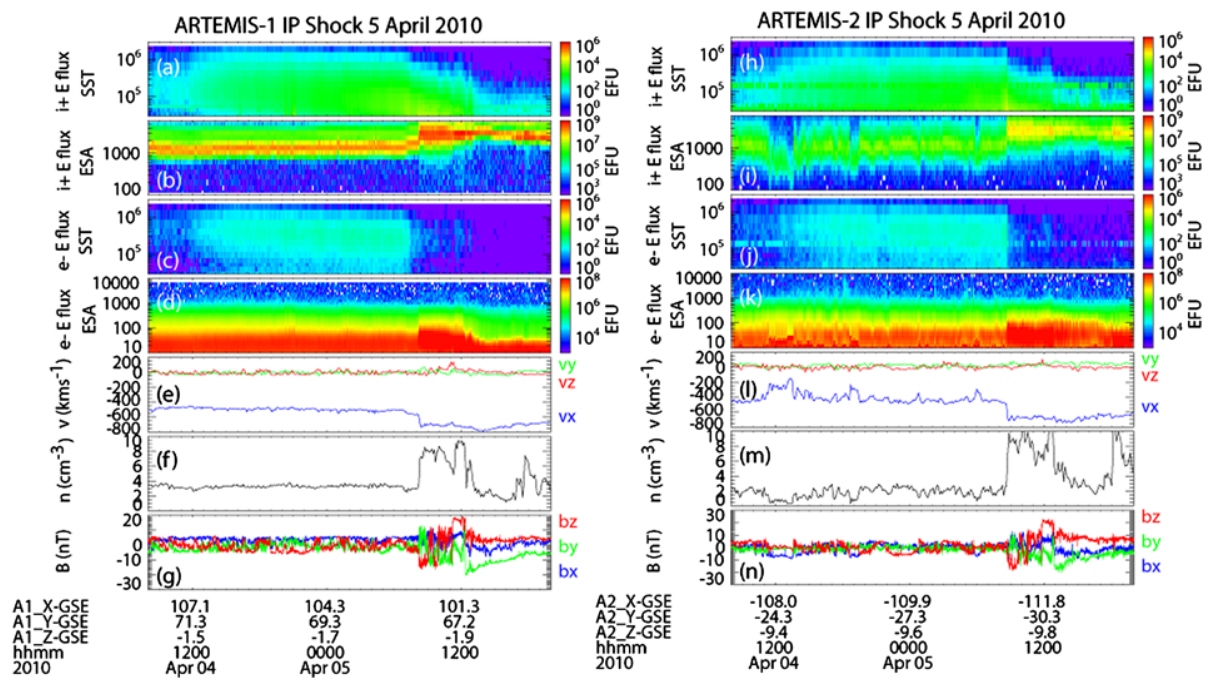

Fig. 11 ARTEMIS P1 (left) and P2 (right) observations of an interplanetary shock on 5 April 2010. From top to bottom, the panels show: SST ion energy flux, ESA ion energy flux, SST electron energy flux, ESA electron energy flux, ESA ion velocity components, ESA ion density, and FGM magnetic field components. Note that because the spacecraft were in transition from Earth to lunar orbit, they were widely separated at this time. The ARTEMIS-1 encounter occurred at 08:12 UT and the ARTEMIS-2 encounter at 08:42 UT. Both ARTEMIS spacecraft detected significant fluxes of energetic electrons and ions 
for tracing the propagation of CMEs through the heliosphere. It is still unclear whether the structuring of type II emissions is due to the curvature of the shock on scales of $10 \mathrm{~s}$ of Earth radii, or due to the curvature of the magnetic field, although it is known that upstream turbulence alone cannot explain the dimensions of the acceleration regions inferred from a single spacecraft (Pulupa and Bale 2008). Furthermore, the ultimate cause of any such rippled shock structures remains unclear, especially as a function of upstream conditions. Since the emissions are controlled by the shock structure, to better understand how type II emission is produced (and thus improve its utility in tracking CMEs) it is necessary to study how shock structure varies with upstream plasma beta, Mach number (also related to the CME speed) and magnetic field orientation.

ARTEMIS measurements will allow investigation of IP shock rippling and inhomogeneities on scales of $0.1-20 R_{\mathrm{E}}$, highly relevant to the problems discussed here (Pulupa and Bale 2008). Two-point measurements of magnetic field vectors will help determine the curvature of magnetic field lines, while two-point measurements of shock normals will help determine shock curvature. Targeted burst mode operations will allow high $(3 \mathrm{~s})$ time resolution 3-D ion and electron distributions to be captured, providing new details about the upstream structure and the presence of electron beams. Burst mode operations can be targeted to capture the interplanetary shock crossing at high time resolution, including the 3-D electric field, to better understand how the electron foreshock beams are created.

\subsubsection{Solar Wind Reconnection}

The recent discovery of reconnection exhausts in the solar wind (Gosling et al. 2005) revealed a new laboratory for reconnection research. Extremely long X-lines, extending hundreds of Earth radii, have been reported in the solar wind (Phan et al. 2006, 2009; Gosling et al. 2007a). All extended X-line events reported so far were found in large magnetic shear current sheets where the reconnection exhausts were wide. However, there are many more low-magnetic shear (and much narrower) reconnection exhausts in the solar wind that can only be resolved by high time resolution plasma measurements (Gosling et al. 2007b). ARTEMIS, in conjunction with Wind, will allow the investigation of the extent of the reconnection X-lines for low magnetic shears, and establish whether their X-line extent scales with current sheet width as suggested in 3D simulations of reconnection (e.g. Shay et al. 2003). The multi-point, high-resolution measurements will also be used to investigate the structure of the reconnection exhaust as a function of the distance from the $\mathrm{X}$-line.

\subsubsection{Solar Wind Turbulence}

Turbulence is a multi-scale phenomenon that mediates the transfer of energy, mass, and momentum. The presence of turbulence within the solar wind has been well established from studies of power spectra, probability distribution functions, scaling exponentials, Reynolds number determinations, etc. Most previous solar wind studies have not focused on the determination of the three fundamental turbulence sale lengths: the correlation scale, the Taylor scale, and the dissipation scale. The correlation scale is associated with the largest possible turbulent eddy scale size. The Taylor scale is the scale size within the inertial range at which viscous damping begins to become important for eddy damping, and the dissipation scale is the scale at which the turbulent eddies have been damped out and the particles are heated. Studies of these fundamental turbulence scales are important to help us understand cosmic ray modulation and particle scattering within the solar wind. 
Studies of magnetic field fluctuations indicate that the correlation scale is on the order of several million kilometers and the Taylor scale on the order of a few thousand kilometers (Mattheaus et al. 1990, 2005; Dasso et al. 2005; Weygand et al. 2007, 2009a, 2009b). However, Mattheus et al. (1990), Dasso et al. (2005), and Weygand et al. (2009a, 2009b) have shown that solar wind turbulence is anisotropic and correlation scale can vary with the respect to the mean magnetic field direction. Mattheaus et al. (1990) attribute this feature to two different types of turbulence present within the solar wind: slab and quasi-two dimensional turbulence. Both Mattheus et al. (1990) and Dasso et al. (2005) used single spacecraft observation to show this anisotropy in the turbulent magnetic field fluctuations and determined the correlation scale by fitting an exponential function to autocorrelation values. Dasso et al. (2005) subdivided results by solar wind speed. They showed that the correlation scale is longest along the mean magnetic field direction in the slow solar wind $\left(<400 \mathrm{~km} \mathrm{~s}^{-1}\right)$ but longest in the perpendicular direction in the fast solar wind $\left(>500 \mathrm{~km} \mathrm{~s}^{-1}\right)$.

Weygand et al. (2009a, 2009b) employed spacecraft pairs within the solar wind to avoid the inherent assumption in previous studies that the solar wind magnetic field fluctuations are frozen into the flow. The Weygand et al. (2009b) study demonstrated systematic variations of the two-dimensional cross-correlation function for slow, intermediate, and fast solar wind speeds. However, two separate exponential fits were required to fit cross-correlation values across the full range of spacecraft separations observed. The first exponential fit the correlation values obtained for large ACE, Geotail, IMP-8, Wind, and Interball-1 interseparation distances, while the second exponential with a smaller decay length fit the much smaller interspacecraft separations for the Cluster spacecraft. Weygand et al. (2009a) hypothesized that this smaller scale resulted from instrumental differences or foreshock turbulence.

However, limited THEMIS solar wind magnetic field measurements indicate that the second exponential decay lengths at all solar wind speed ranges do not result from instrumental bias or foreshock turbulence, but rather represent real features in the solar wind. Figure 12 (Weygand et al. 2009b) displays the cross correlation values versus the spacecraft separation for various spacecraft pairs in the fast solar wind $(>600 \mathrm{~km} / \mathrm{s})$. The blue values between the two vertical dashed lines are the cross-correlation values from the THEMIS B and C spacecraft.

Fig. 12 Cross-correlation coefficients versus the distance separating spacecraft for the fast solar wind $(>600 \mathrm{~km} / \mathrm{s})$ outside the foreshock. Cluster correlations cover the range of separations between 0 and $\sim 10,000 \mathrm{~km}$ in black. ACE, Wind, Geotail, IMP8, and Interball-1 correlations cover separations greater than $100,000 \mathrm{~km}$. Blue asterisks indicate limited THEMIS B and $\mathrm{C}$ correlations between the two dashed vertical lines. The range curve results from a single exponential fit to all the data. The dashed curve represents the sum of two exponentials fit to all the data

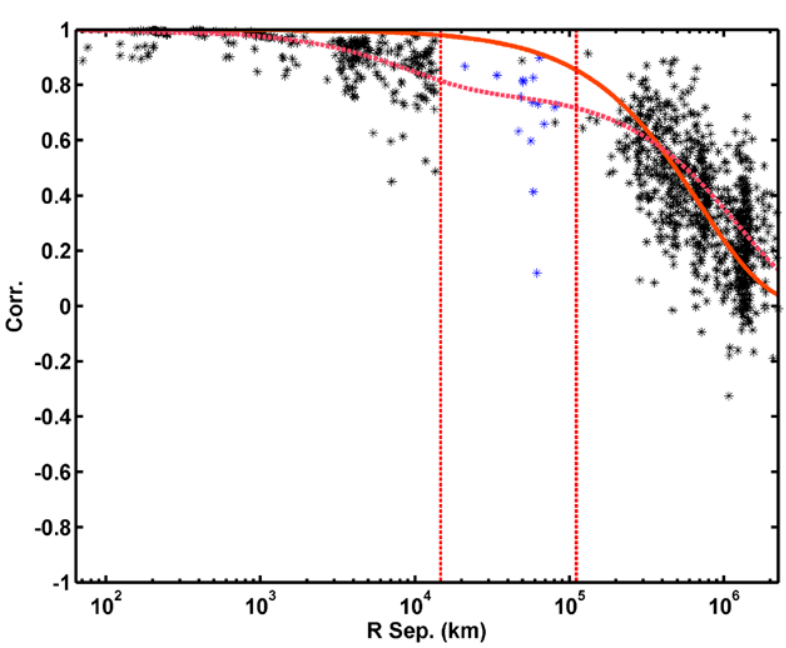


While the THEMIS cross correlation values have been an enormous help, the physical meaning of the second exponential decay lengths remains unclear. It may be related to the hypothesis that turbulence is bound within solar wind flux tubes (Borovsky 2008). Large decay lengths are related to the walls of the solar wind flux tubes while the small decay lengths are associated with the correlation scale of the magnetic field turbulence within the flux tubes.

ARTEMIS will play a critical role in these investigations. Inter-separation distances at the Moon will range from $500 \mathrm{~km}$ to about $31,000 \mathrm{~km}$, precisely filling in the poorly sampled region from $1.5 \times 10^{4}$ to $3.0 \times 10^{4} \mathrm{~km}$ where Fig. 12 indicates a transition between the two exponential fits.

\subsubsection{Upstream Monitors}

In lunar orbit, ARTEMIS will be an excellent monitor of the solar wind conditions upstream from the Earth's magnetosphere. Unlike Earth-orbiting satellites, ARTEMIS will remain outside the magnetosphere for extended periods of time, and will, for example, be able to observe the entire passage of a CME. As such, ARTEMIS will provide useful input to global simulations of the Earth's magnetosphere and services such as the Community Coordinated Modeling Center (CCMC). ARTEMIS will be closer to the Earth than existing L1 solar wind monitors, and therefore offers a more precise measurement of the exact solar wind input to the magnetosphere-such measurements will be of use to other teams studying the magnetosphere with both satellite- and ground-based experiments. Finally, by comparing ARTEMIS data to that from L1 monitors such as ACE and Wind, it will be possible to develop new models that advect L1 solar wind monitoring data to the Earth, thereby improving our understanding of how L1 monitoring data should be used to predict magnetospheric dynamics.

\subsection{The Lunar Wake}

As an essentially non-magnetic and non-conducting body with no ionosphere, the Moon absorbs most of the incident solar wind plasma, leaving a plasma void, or wake, within plasma flows (Schubert and Lichtenstein 1974). The plasma cavity formed downstream from the Moon represents one of the best natural plasma vacuums in the solar system, and an excellent basic physics laboratory for understanding the general process of plasma expansion into a vacuum, with applications ranging from low-altitude earth orbiting satellites to outer planet moons. Although the lunar wake has been studied since the Apollo era (Ness et al. 1967; Colburn et al. 1967), we still do not fully understand many aspects of its formation, dynamics, and refilling.

Nevertheless, our understanding of the wake has advanced with each new lunar mission. Far from being a passive region, the wake hosts a wide variety of dynamic phenomena (see Fig. 13). Wind, with a suite of modern plasma instruments, made a number of lunar flybys and discovered a wake extending to at least $25 R_{\mathrm{L}}$ downstream (Clack et al. 2004), counter-streaming anisotropic ion beams refilling the wake along magnetic field lines (Ogilvie et al. 1996; Clack et al. 2004), and electrostatic and electromagnetic plasma waves in and around the wake (Kellogg et al. 1996; Farrell et al. 1996; Bale et al. 1997). Nozomi observed non-thermal ions and counter-streaming electrons upstream from the Moon (Futaana et al. 2001, 2003), possibly associated with wake processes. Geotail observed ULF waves associated with the lunar wake region (Nakagawa et al. 2003). Lunar Prospector discovered the important role of non-Maxwellian solar wind electrons in driving low-altitude wake refilling (Halekas et al. 2005). Kaguya, Chang'E, and Chandrayaan discovered ion scattering from the dayside surface (Saito et al. 2010; Wieser et al. 


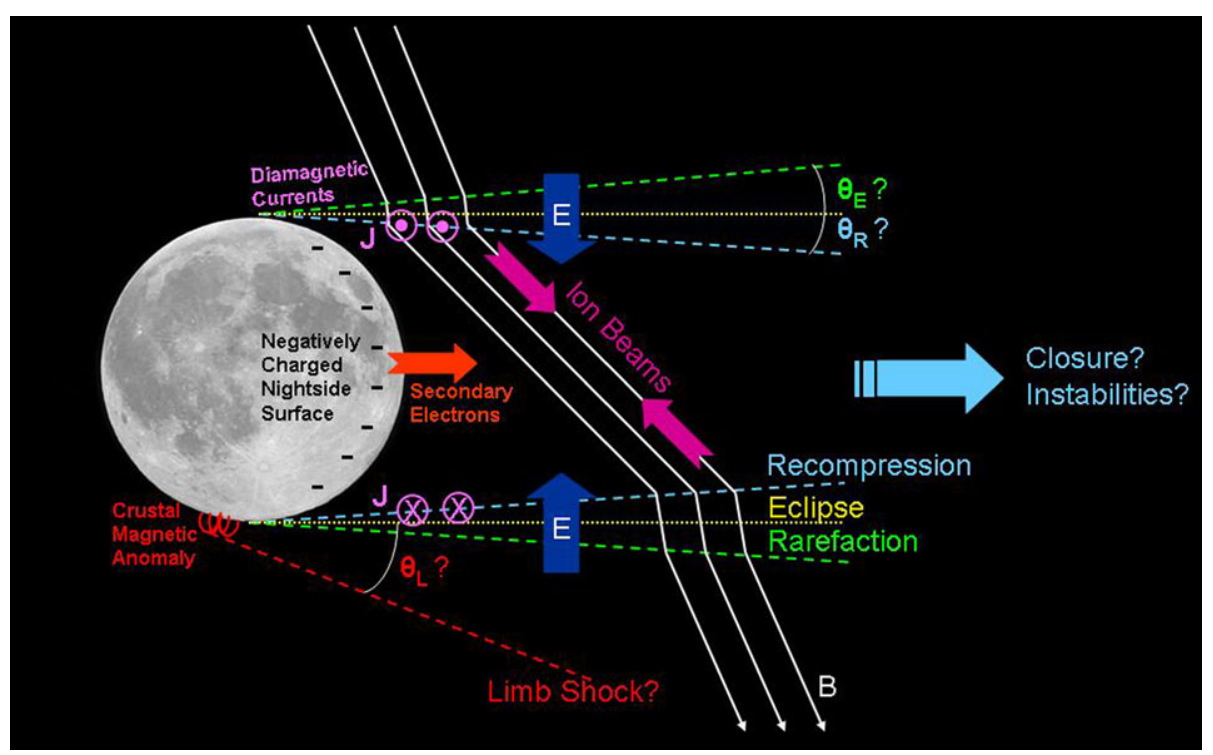

Fig. 13 Our current understanding of the electrodynamics of the Moon's interaction with the solar wind. The Moon carves out a cavity, known as the wake, in the oncoming solar wind. Ion beams stream inward to fill the cavity, which is bounded by rarefaction waves, diamagnetic currents, and inward-pointing electric fields. Crustal magnetic anomalies may launch shocks into the solar wind, while the nightside lunar surface charges negative and emits secondary electrons

2009), and observed gyrating protons (Type-I entry) and re-picked up scattered protons (Type-II entry) refilling the low altitude wake perpendicular to magnetic field lines (Nishino et al. 2009a, 2009b; Holmstrom et al. 2010; Wang et al. 2010).

While these observations have shed light on the global structure of the lunar wake and emphasized the need to treat it kinetically, our understanding of the physical nature of the wake and how it refills is limited primarily due to the relative paucity of in situ measurements made in the region. Early Explorer and Apollo satellites carried limited plasma instrumentation and the Lunar Prospector instrument package did not include ion detectors or electric field analyzers. Wind provided a relatively complete plasma data set from the lunar wake, but made only a handful of passes, leaving wake coverage far from complete. Nozomi and Geotail also have made only a very limited number of lunar flybys. Chandrayaan, Chang'E, and Kaguya only observed the wake over narrow ranges of distances from the Moon. To date, there has not been a dedicated mission to provide comprehensive coverage of the lunar wake over a wide range of distances and complete our understanding of its structure.

\subsubsection{Structure of the Wake}

Global hybrid simulations (kinetic ions, fluid electrons) confirm that kinetic effects are important. Figure 14, showing the formation of a plasma void in the lunar wake that is refilled by two beams counter-streaming along magnetic field lines in $X-Y$ plane, demonstrates the complex structure of the lunar wake even for ambient magnetic field orientations that lie transverse to the wake axis. The same simulation also indicates that the lunar wake could extend well beyond $25 R_{\mathrm{L}}$ downstream. 


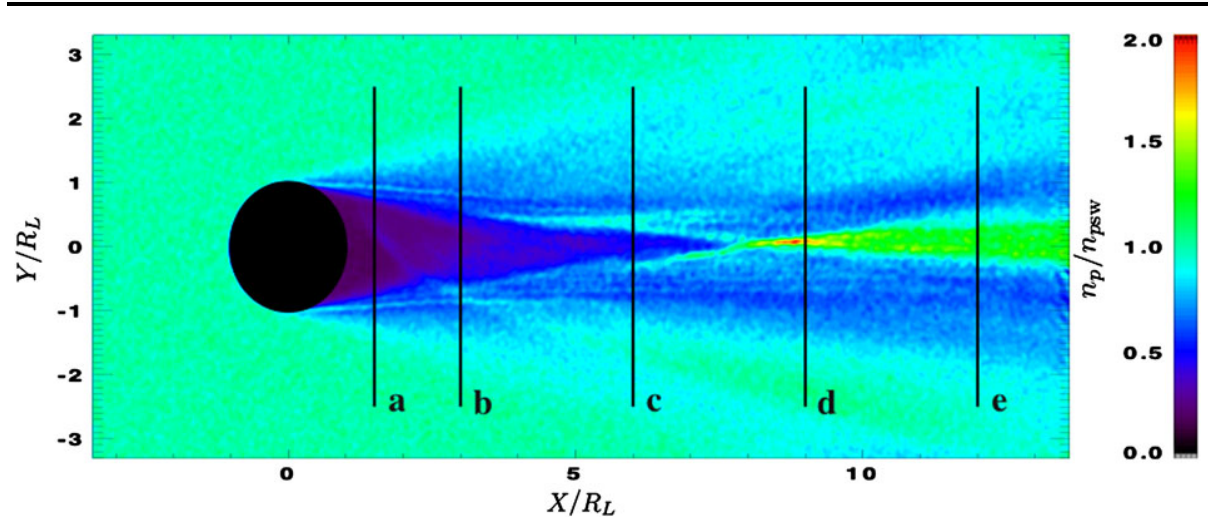

Fig. 14 Results from a global hybrid simulation for the interaction of the solar wind with the Moon. Proton densities, normalized to the value in the unperturbed solar wind, are shown in the $X-Y$ plane containing the IMF, which points in the $Y$-direction. The results show the formation of a plasma void in the lunar wake that is refilled by two beams counter-streaming along magnetic field lines

As illustrated in Fig. 13, asymmetries in the lunar wake structure are expected whenever the magnetic field does not lie parallel to the wake, since diamagnetic current systems, and the resulting magnetic field perturbations, differ for perpendicular vs. parallel magnetic fields. In addition, limb compressions are often observed external to the wake cavity and rarefaction wave, located downstream from crustal magnetic anomalies (Russell and Lichtenstein 1975). Previous measurements suggest that these structures are compressional features (Siscoe et al. 1969) that propagate outward at magnetosonic wave velocities (Whang and Ness 1970). One expects them to propagate downstream from crustal magnetic field regions, producing a highly asymmetric structure external to the main wake cavity. However, no observations have clearly confirmed this supposition, or determined how far downstream these features propagate and to what degree they affect the structure of the wake interior to them. Many observations have found asymmetric wake characteristics on individual orbits (Ness et al. 1968), but single spacecraft cannot clearly distinguish between asymmetries and temporal variations.

The orbital coverage and complete plasma instrumentation of ARTEMIS will enable a comprehensive determination of the wake's extent and structure. The two-point measurements provided by ARTEMIS will allow unambiguous identification of asymmetries in the wake due to the perturbing influences of solar wind and crustal magnetic fields or other effects.

\subsubsection{Wake Refilling and Dynamics}

Previous missions have measured several modes of wake refilling, but we do not yet understand their relative importance or the interplay between them. Wind saw ion beams accelerated along magnetic field lines into the wake from the flanks (Ogilvie et al. 1996; Clack et al. 2004) several lunar radii downstream, implying a potential drop across the wake boundary that occurs as a natural consequence of the pressure gradient across the wake boundary and the difference in electron and ion thermal velocities. Simulations have contributed greatly to our understanding of this process, which refills the wake along magnetic field lines (Farrell et al. 1998; Birch and Chapman 2001, 2002; Kallio 2005; Travnicek et al. 2005; Kimura and Nakagawa 2008). Meanwhile, recent low altitude observations show that 
the wake also refills perpendicular to magnetic field lines (Nishino et al. 2009a, 2009b; Holmstrom et al. 2010; Wang et al. 2010). Only ARTEMIS, with its elliptical orbit, can unravel the interplay between parallel and perpendicular refilling processes, and determine their relative importance as a function of location in the wake.

The wake provides a rich laboratory for plasma waves and instabilities, with a broad spectrum of waves observed in the central wake (Kellogg et al. 1996), and even far upstream on magnetic field lines connected to the wake boundary (Nakagawa et al. 2003; Farrell et al. 1996; Bale et al. 1997). Waves observed to date include ion acoustic waves and Langmuir waves from instabilities related to differential ion/electron shadowing (Bale et al. 1997), and whistlers produced by beam instabilities near the wake boundary (Nakagawa et al. 2003; Farrell et al. 1996, 2008). Waves predicted but not conclusively observed before ARTEMIS include instabilities generated by counter-streaming electrons in the central wake (Birch and Chapman 2001, 2002), ion acoustic-like interactions generated by refilling ion beams in the central wake (Farrell et al. 1998), and low frequency electromagnetic turbulence with frequencies near the local proton gyrofrequency (Travnicek et al. 2005). Most recently, Kaguya has observed broad spectrum electrostatic turbulence associated with a two-stream instability formed by electrons pulled into the wake along field lines by the superabundance of charge from pickup ion Type II entry perpendicular to magnetic field lines (Nishino et al. 2010). We have barely explored this menagerie of plasma waves, and the instability growth mechanisms and wave-particle interactions remain far from understood. As illustrated in Fig. 15, the first lunar wake flyby by ARTEMIS revealed electrostatic oscillations associated with counter-streaming electrons in the central wake and the regions magnetically connected to it, providing the first confirmation for the predictions of Nakagawa et al. (2003) and Birch and Chapman (2001, 2002). See Halekas et al. (2011, this

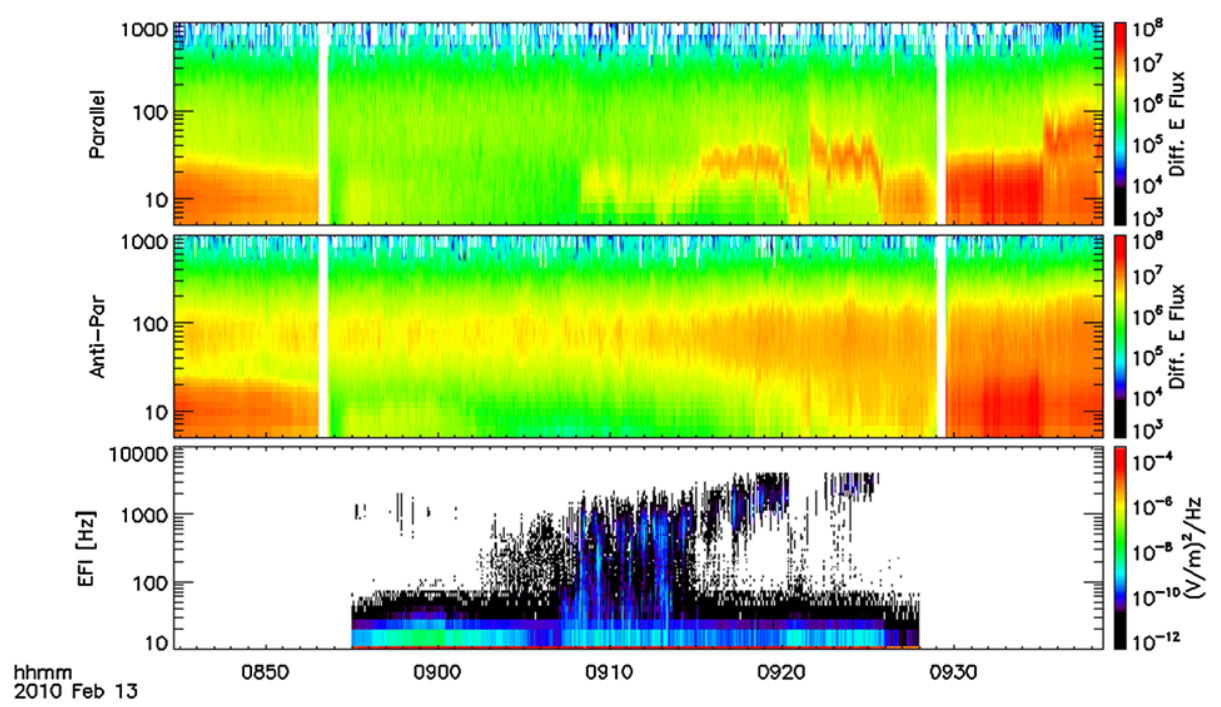

Fig. 15 Observations from the first lunar wake flyby by ARTEMIS probe P1 on Feb 13, 2010. The figure shows energy-time spectrograms for electrons streaming parallel $\left(0-15^{\circ}\right.$ pitch angle) and anti-parallel $\left(165-180^{\circ}\right)$ to the magnetic field (in $\mathrm{eV} /\left(\mathrm{cm}^{2} \mathrm{~s} \mathrm{sreV}\right)$ ), and an electric field wave frequency-time spectrogram. The anti-parallel streaming solar wind strahl population penetrates all the way through the wake from the exit side, while most of the core population is excluded from the central wake by the wake potential. A residual core population penetrates from the entry side of the wake, and is accelerated outward by the wake potential, forming a counter-streaming distribution that excites electrostatic oscillations 
issue) for more details. Further passes will enable researchers to determine the distribution of wave activity within the wake.

\subsubsection{Response to External Drivers}

The wake structure will certainly vary in dynamic response to external drivers. Several studies (Ogilvie and Ness 1969; Colburn et al. 1971; Whang and Ness 1972; Halekas et al. 2005) provide tantalizing hints how the wake responds to changing solar wind conditions, but the limited data and orbital coverage of the previous missions constrains our knowledge of this response to external drivers. Hybrid simulations have shown that the orientation of the IMF with respect to the solar wind flow direction affects both the downstream extent and symmetry of the lunar wake (Travnicek et al. 2005).

Although the Moon spends the majority of its time in the solar wind, it also spends $\sim 5$ days each month in the terrestrial magnetosphere, where the plasma environment differs greatly from that in the solar wind. Magnetic field configurations and flow speeds in the plasma sheet are highly variable, which should result in lunar wakes very different from those in the solar wind. For example, reconnection beyond $60 R_{\mathrm{E}}$ should result in sunward plasma sheet plasma flows and a sunward-orientated wake.

Using dual-probe measurements, ARTEMIS will determine the response of the lunar wake to solar wind drivers. ARTEMIS will provide the first detailed measurement of the lunar wake plasma environment within the terrestrial magnetosphere.

\section{Concluding Remarks}

From vantage points near and in orbit about the Moon, ARTEMIS will provide the comprehensive two-point particle and electromagnetic field observations needed to chart and understand a host of planetary phenomena. Observations of pick-up ions will be used to determine the composition of the exosphere and hence the lunar surface as a function of external conditions. Electric fields that can loft dust in the vicinity of the Moon will be measured both directly and remotely. The structure of crustal magnetic anomalies that shield some regions of the Moon while creating mini-magnetospheres complete with bow shocks in the oncoming solar wind will be mapped.

The same lunar vantage points offer an opportunity to address a series of longstanding heliospheric research problems. ARTEMIS will map the structure of the Earth's magnetotail, determining its structure and the characteristics of magnetic reconnection, particle energization, and turbulence as function of solar wind conditions. While in the solar wind, the two spacecraft will provide the observations needed to understand reconnection at interplanetary discontinuities and particle acceleration at both the Earth's bow shock and interplanetary shocks. They will also serve as excellent monitors of the solar wind input into the magnetosphere for other forthcoming NASA and international missions. Finally, the ARTEMIS spacecraft will provide the comprehensive observations needed to understand the steadystate and time-dependent structure of and processes within the lunar wake.

The novel repurposing of the outermost two THEMIS spacecraft demonstrates the creativity of the THEMIS/ARTEMIS team at its best. It has set the stage for potentially dramatic advances in our understanding of the Moon and its environment. This will be a joint undertaking of both the THEMIS/ARTEMIS team and the international community, for all of the observations returned by ARTEMIS will become immediately available via the mission's web site, themis.ssl.Berkeley.edu, just as is the case for THEMIS. 
Acknowledgements Work at UCB and UCLA was supported by NASA Contract NAS5-02099. Work at NASA/GSFC was supported by THEMIS MO\&DA. J.P.E. is supported by an STFC Advanced Fellowship at Imperial College.

\section{References}

K.A. Anderson et al., Space Sci. Instrum. 1, 439 (1975)

V. Angelopoulos et al., J. Geophys. Res. 97, 4027 (1992). doi:10.1029/91JA02701

V. Angelopoulos et al., J. Geomagn. Geoelectr. 48, 629 (1996)

V. Angelopoulos, Space Sci. Rev. 141 (2008). doi:10.1007/s11214-008-9336-1

V. Angelopoulos, Space Sci. Rev. (2011) (this issue). doi:10.1007/s11214-010-9687-2

W.I. Axford, C.O. Hines, Can. J. Phys. 39, 1433 (1961)

I.A. Axford et al., in Proceedings of the 15th International Cosmic Ray Conf., vol. 11 (1977), p. 132

S.D. Bale et al., Geophys. Res. Lett. 24, 1427 (1997)

S.D. Bale et al., Geophys. Res. Lett. 26, 1573 (1999)

W. Baumjohann et al., J. Geophys. Res. 94, 6597 (1989). doi:10.1029/JA094iA06p06597

W. Baumjohann et al., J. Geophys. Res. 95, 3801 (1990). doi:10.1029/JA095iA04p03801

A.R. Bell, Mon. Not. R. Astron. Soc. 182, 147 (1978a)

A.R. Bell, Mon. Not. R. Astron. Soc. 182, 443 (1978b)

P.C. Birch, S.C. Chapman, Phys. Plasmas 8, 4551 (2001)

P.C. Birch, S.C. Chapman, Phys. Plasmas 9, 1785 (2002)

R.D. Blandford, J.P. Ostriker, Astrophys. J. 221, L29 (1978)

J.E. Borovsky, J. Geophys. Res. 113 (2008). doi:10.1029/2007JA012684

T.K. Breus et al., Adv. Space Res. 36, 2043 (2005)

D. Burgess et al., Space Sci. Rev. 118, 205 (2005)

D. Burgess, Lect. Notes Phys. 725, 161 (2007)

C.C. Chaston et al., Phys. Rev. Lett. 99, 175004 (2007)

C.C. Chaston et al., Geophys. Res. Lett. 35 (2008). doi:10.1029/2008GL033601

S.-H. Chen, M.G. Kivelson, Geophys. Res. Lett. 20, 2699 (1993)

S.M. Cisowski et al., J. Geophys. Res. Suppl. 88, A691 (1983)

D. Clack et al., Geophys. Res. Lett. 31 (2004). doi:10.1029/2003GL018298

J.B. Cladis et al., J. Geophys. Res. 99, 53 (1994)

D.S. Colburn et al., Science 158, 1040 (1967)

D.S. Colburn et al., J. Geophys. Res. 76, 2940 (1971)

P.J. Coleman et al., Proc. Lunar Sci. Conf. 3, 2271 (1972)

J.E.P. Connerney et al., Science 284, 794 (1999)

J.E.P. Connerney et al., Space Sci. Rev. 1, 1 (2004)

D.R. Criswell, in Photon and Particle Interaction in Space, ed. by R.J.L. Grard (Reidel, Dordrecht, 1973), p. 545

S. Dasso et al., Astrophys. J. 635, L181 (2005)

G. Delory et al., in $40^{\text {th }}$ LPSC, id. 2025, 2009

M. Delva et al., Geophys. Res. Lett. 35 (2008). doi:10.1029/2007GL032594

M.I. Desai et al., J. Geophys. Res. 105, 61 (2000)

M. Dougherty et al., Science 311, 1406 (2006)

J.F. Drake et al., Nature 443, 553 (2006). doi:10.1038/nature05116

J.F. Drake et al., J. Geophys. Res. 114 (2009). doi:10.1029/2008JA013701

J.W. Dungey, Phys. Rev. Lett. 6, 47 (1961)

Dyal, et al., Rev. Geophys. Space Phys. 12, 568 (1974)

J.P. Eastwood et al., Space Sci. Rev. 118, 41 (2005)

D.H. Fairfield et al., J. Geophys. Res. 105, 21159 (2000)

W.M. Farrell et al., Geophys. Res. Lett. 23, 1271 (1996)

W.M. Farrell et al., J. Geophys. Res. 103, 23653 (1998)

W.M. Farrell et al., Geophys. Res. Lett. 34 (2007). doi:10.1029/2007GL029312

W.M. Farrell et al., Geophys. Res. Lett. 35 (2008). doi:10.1029/2007GL032653

M.H. Farris, C.T. Russell, J. Geophys. Res. 99, 17681 (1994)

J.A. Fedder, J.G. Lyon, J. Geophys. Res. 100, 3623 (1995)

W.C. Feldman et al., J. Geophys. Res. 90, 233 (1985)

B. Flynn, M. Mendillo, Science 261, 184 (1993)

H.U. Frey et al., Nature 426, 533 (2003) 
M. Fujimoto, T. Terasawa, J. Geophys. Res. 99, 8601 (1994)

M. Fuller, Rev. Geophys. Space Phys. 12, 23 (1974)

M. Fuller, S. Cisowski, in Geomagnetism, vol. 2, ed. by J. Jacobs (Academic Press, London, 1987), p. 307

Y. Futaana et al., J. Geophys. Res. 106, 18729 (2001)

Y. Futaana et al., J. Geophys. Res. 108, 1025 (2003). doi:10.1029/2002JA009366

V.L. Galinsky, B.U.Ö. Sonnerup, Geophys. Res. Lett. 21, 2247 (1994)

J. Giacalone, Astrophys. J. 609, 452 (2004)

G.R. Gladstone et al., LPSC, abstract 2277 (2010)

T.I. Gombosi et al., in Physics of Space Plasmas, ed. by T. Chang, J.R. Jasperse (MIT, Cambridge, 1998), p. 121

T.I. Gombosi et al., J. Geophys. Res. 105, 13141 (2000)

J.T. Gosling et al., J. Geophys. Res. 94, 3555 (1989)

J.T. Gosling et al., J. Geophys. Res. 110 (2005). doi:10.1029/2004JA010809

J.T. Gosling et al., Geophys. Res. Lett. 34 (2007a). doi:10.1029/2006GL029033

J.T. Gosling et al., Geophys. Res. Lett. 34 (2007b). doi:10.1029/2007GL030706

F.S. Grant, G.F. West, Interpretation Theory in Applied Geophysics (McGraw-Hill, New York, 1965)

J.M. Grebowsky et al., Adv. Space Res. 33, 176 (2004)

R.E. Grimm, NRC planetary science white paper. NRC, Washington, DC, http://www.psi.edu/decadal/ 2009

R.E. Grimm, G.T. Delory, NLSI Lunar Science Conf., abstract \#2075 (2008)

R.E. Grimm, H.Y. McSween, $40^{\text {th }}$ LPSC XL, abstract \#1958 (2009)

P.N. Guzdar et al., J. Geophys. Res. 106, 275 (2001)

J.S. Halekas, PhD thesis, UC Berkeley, 2003

J.S. Halekas et al., J. Geophys. Res. 106, 27841 (2001)

J.S. Halekas et al., Geophys. Res. Lett. 29 (2002a). doi:10.1029/2001GL014428

J. Halekas et al., Geophys. Res. Lett. 29 (2002b). doi:10.1029/2001GL013924

J.S. Halekas et al., J. Geophys. Res. 110 (2005). doi:10.1029/2004JA010991

J.S. Halekas et al., Geophys. Res. Lett. 33 (2006a). doi:10.1029/2006GL025931

J.S. Halekas et al., Geophys. Res. Lett. 33 (2006b). doi:10.1029/2006GL027684

J.S. Halekas et al., Geophys. Res. Lett. 34 (2007). doi:10.1029/2006GL028517

J.S. Halekas et al., Planet. Space Sci. 56, 941 (2008a). doi:10.1016/j.pss.2008.01.008

J.S. Halekas et al., J. Adv. Space Res. 41, 1319 (2008b). doi:10.1016/j.asr.2007.04.003

J.S. Halekas et al., J. Geophys. Res. 113 (2008c). doi:10.1029/2008JA013194

J.S. Halekas et al., Space Sci. Rev. (2011) (this issue). doi:10.1007/s11214-010-9738-8

M. Hapgood, Ann. Geophys. 25, 2037 (2007). doi:10.5194/angeo-25-2037-2007

E.M. Harnett, R. Winglee, J. Geophys. Res. 105, 24997 (2000)

E.M. Harnett, R. Winglee, J. Geophys. Res. 108 (2003). doi:10.1029/2002JA009617

E.C. Hartle, R. Killen, Geophys. Res. Lett. 33 (2006). doi:10.1029/2005GL024520

E.C. Hartle, E.C. Sittler Jr., J. Geophys. Res. 112 (2007). doi:10.1029/2006JA012157

E.C. Hartle, G.E. Thomas, J. Geophys. Res. 79, 1519 (1974)

H. Hasegawa et al., Ann. Geophys. 22, 1251 (2004)

H. Hasegawa et al., Adv. Space Res. 36, 1772 (2005)

H. Hasegawa et al., J. Geophys. Res. 111 (2006). doi:10.1029/2006JA011728

H. Hasegawa et al., Geophys. Res. Lett. 35 (2008). doi:10.1029/2008GL034767

M. Hilchenbach et al., in Proceedings of Solar Wind Seven, ed. by E. Marsch, G. Schwenn (Pergamon Press, Oxford, 1991)

M. Hilchenbach et al., Adv. Space Res. 13, 321 (1993)

M.M. Holmström et al., J. Geophys. Res., in press (2010). doi:10.1029/2009JA014843

L.L. Hood, J. Geophys. Res. 97, 18275 (1992)

L.L. Hood, N.A. Artemieva, Icarus 193, 485 (2008)

L.L. Hood, G. Schubert, Science 204, 49 (1980)

L.L. Hood, C.P. Sonett, Geophys. Res. Lett. 9, 37 (1982)

L.L. Hood, Z. Huang, J. Geophys. Res. 96, 9837 (1991)

L.L. Hood, R.W. Williams, in LPSC 19 (1989), p. 19

L.L. Hood et al., J. Geophys. Res. 86, 1055 (1981)

L.L. Hood et al., J. Geophys. Res. 87, 5311 (1982)

L.L. Hood et al., Geophys. Res. Lett. 26, 2327 (1999)

L.L. Hood et al., J. Geophys. Res. 106, 27825 (2001)

M. Hoshino et al., J. Geophys. Res. 106, 25979 (2001). doi:10.1029/2001JA900052

A. Ieda et al., J. Geophys. Res. 103, 4453 (1998)

ILN, Final Report of the Science Definition Team for the ILN Anchor Nodes. NASA, Washington, DC, 2009, http://lunarscience.arc.nasa.gov/pdf/ILN_Final_Report.pdf 
E. Kallio, Geophys. Res. Lett. 32 (2005). doi:10.1029/2004GL021989

P.J. Kellogg et al., Geophys. Res. Lett. 23, 1267 (1996)

C.F. Kennel et al., J. Geophys. Res. 91, 11917 (1986)

K.K. Khurana et al., Nature 395, 777 (1998)

S. Kimura, T. Nakagawa, Earth Planets Space 60, 591 (2008)

E. Kirsch et al., Adv. Space Res. 20, 845 (1997)

A. Kis et al., Geophys. Res. Lett. 31 (2004). doi:10.1029/2004GL020759

M.G. Kivelson et al., Adv. Space Res. 16, 59 (1995)

M.G. Kivelson et al., Science 274, 396 (1996)

E.A. Kronberg et al., J. Geophys. Res. 114 (2009). doi:10.1029/2008JA013754

S.H. Lai, L.H. Lyu, J. Geophys. Res. 111 (2006). doi:10.1029/2004JA010724

M.A. Lee, J. Geophys. Res. 88, 6109 (1983)

W. Li et al., J. Geophys. Res. 113 (2008). doi:10.1029/2007JA012604

R.P. Lin et al., J. Geophys. Res. 79, 489 (1974)

R.P. Lin et al., Proc. Lunar Sci. Conf. 7, 2691 (1976)

R.P. Lin et al., Icarus 74, 529 (1988)

R.P. Lin et al., Science 281, 1480 (1998)

J.E. McCoy, Proc. Lunar Sci. Conf. 7, 1087 (1976)

J.E. McCoy, Criswell, Proc. Lunar Sci. Conf. 5, 2991 (1974)

U. Mall et al., Geophys. Res. Lett. 25, 3799 (1998)

R.H. Manka, in Photon and Particle Interactions with Surfaces in Space, ed. by R.J.L. Grard (Reidel, Dordrecht, 1973), p. 347

W.H. Matthaeus et al., J. Geophys. Res. 95, 20673 (1990)

W.H. Matthaeus et al., Phys. Rev. Lett. 95, 231101 (2005)

Y. Matsumoto, K. Seki, J. Geophys. Res. 112 (2007). doi:10.1029/2006JA012114

M. Mendillo et al., Geophys. Res. Lett. 18, 2907 (1991)

M. Mendillo et al., Icarus 137, 13 (1999)

D.L. Mitchell et al., Icarus 194, 401 (2008). doi:10.1016/j.icarus.2007.10.027

A. Miura, J. Geophys. Res. 89, 801 (1984)

A. Miura, J. Geophys. Res. 97, 10655 (1992)

A. Miura, Phys. Plasmas 4, 2871 (1997)

E. Möbius et al., Geophys. Res. Lett. 13, 1372 (1986)

T. Nakagawa et al., Earth Planets Space 55, 569 (2003)

T.K.M. Nakamura et al., Phys. Plasmas 17, 042119 (2010)

N.F. Ness et al., J. Geophys. Res. 72, 5769 (1967)

N.F. Ness et al., J. Geophys. Res. 73, 3421 (1968)

P.T. Newell et al., J. Geophys. Res. 102, 127 (1997)

A. Nishida et al., Geophys. Res. Lett. 22, 2453 (1995)

M.N. Nishino et al., Geophys. Res. Lett. 36 (2009a). doi:10.1029/2009GL039049

M.N. Nishino et al., Geophys. Res. Lett. 36 (2009b). doi:10.1029/2009GL039444

M.N. Nishino et al., Geophys. Res. Lett. 37 (2010). doi:10.1029/2010GL043948

M.N. Nishino et al., Planet. Space Sci. 59 (2011). doi:10.1016/j.pss.2010.03.011

T. Nitter et al., J. Geophys. Res. 103, 6605 (1998)

K.W. Ogilvie, N.F. Ness, J. Geophys. Res. 74, 4123 (1969)

K.W. Ogilvie et al., Geophys. Res. Lett. 10, 1255 (1996)

T. Ogino et al., IEEE Trans. Plasma Sci. 20, 817 (1992)

M. Øieroset et al., J. Geophys. Res. 105, 25247 (2000)

M. Øieroset et al., Phys. Rev. Lett. 89 (2002). doi:10.1103/PhysRevLett.89.195001

M. Øieroset et al., Geophys. Res. Lett. 31 (2004). doi:10.1029/2004GL020321

M. Øieroset et al., J. Geophys. Res. 113 (2008). doi:10.1029/2007JA012679

N. Omidi et al., J. Geophys. Res. 107, 1487 (2002). doi:10.1029/2002JA009441

W.R. Paterson et al., J. Geophys. Res. 104, 22779 (1999)

T.-D. Phan et al., Nature 439, 175 (2006)

T.-D. Phan et al., Geophys. Res. Lett. 36 (2009). doi:10.1029/2009GL037713

A. Poppe, M. Horányi, J. Geophys. Res. 115 (2010). doi:10.1029/2010JA015286

A.E. Potter, T.H. Morgan, Science 241, 675 (1988)

M. Pulupa, S.D. Bale, Astrophys. J. 676, 1330 (2008)

J. Raeder, J. Geophys. Res. 104, 17357 (1999)

J. Raeder, J. Geophys. Res. 105, 13149 (2000)

J. Raeder, Ann. Geophys. 24, 381 (2006)

J. Raeder et al., Geophys. Res. Lett. 22, 349 (1995) 
J.J. Rennilson, D.R. Criswell, Moon 10, 121 (1974)

N.C. Richmond et al., J. Geophys. Res. 110 (2005). doi:10.1029/2005JA002405

C.T. Russell, R.C. Elphic, Space Sci. Rev. 22, 681 (1978)

C.T. Russell, X. Blanco-Cano, J. Atmos. Sol.-Terr. Phys. 69, 1723 (2007)

C.T. Russell, B.R. Lichtenstein, J. Geophys. Res. 80, 4700 (1975)

C.T. Russell et al., in LPSC $12^{\text {th }}$ (Pergamon, New York, 1982), p. 831

C.T. Russell et al., Geophys. Res. Lett. 17, 897 (1990)

A.E. Saal et al., Nature 454, 192 (2008)

Y. Saito et al., Geophys. Res. Lett. 35 (2008). doi:10.1029/2008GL036077

Y. Saito et al., Space Sci. Rev. 154 (2010). doi:10.1007/s11214-010-9647-x

E.T. Sarris et al., J. Geophys. Res. 92, 12083 (1987)

G. Schubert, B.R. Lichtenstein, Rev. Geophys. Space Phys. 12, 592 (1974)

G. Schubert, K. Schwartz, J. Geophys. Res. 77, 76 (1972)

A. Schuster, H. Lamb, Philos. Trans. R. Soc. A 180, 467 (1889)

M.A. Shay et al., Geophys. Res. Lett. 30, 1345 (2003). doi:10.1029/2002GL016267

D.G. Sibeck, G.L. Siscoe, J. Geophys. Res. 89, 10709 (1984)

D.G. Sibeck et al., J. Geophys. Res. 90, 4011 (1985)

D.G. Sibeck et al., in Magnetotail Physics, ed. by A.T.Y. Lui (Johns Hopkins Press, Baltimore, 1987), p. 7

F. Simpson, K. Bahr, Practical Magnetotellurics (Cambridge University Press, Cambridge, 2005)

G.L. Siscoe et al., J. Geophys. Res. 74, 59 (1969)

J.A. Slavin et al., Geophys. Res. Lett. 26, 2897 (1999)

C.P. Sonett et al., Proc. Lunar Sci. Conf 3rd 2, 2309 (1972)

C.P. Sonett, Rev. Geophys. Space Phys. 20, 411 (1982)

D.J. Southwood, Planet. Space Sci. 16, 587 (1968)

S.A. Stern, Rev. Geophys. 37, 453 (1999)

T.J. Stubbs et al., Adv. Space Res. 37, 59 (2006)

T.J. Stubbs et al., in Dust in Planetary Systems, vol. SP-643, ed. by H. Krüger, A.L. Graps (ESA, Noordwijk, 2007), p. 181

K. Takagi et al., J. Geophys. Res. 111 (2006). doi:10.1029/2006JA011631

T. Tanaka et al., Geophys. Res. Lett. 22 (2009). doi:10.1029/2009GL040682

T. Terasawa, Adv. Space Res. 15, 53 (1995)

T. Terasawa, Prog. Theor. Phys. Suppl. 151, 95 (2003)

K.J. Trattner et al., J. Geophys. Res. 99, 13389 (1994)

P. Travnicek et al., Geophys. Res. Lett. 32 (2005). doi:10.1029/2004GL022243

A.L. Tyler et al., Geophys. Res. Lett. 15, 1141 (1988)

A. Usadi et al., J. Geophys. Res. 98, 7503 (1993)

P. Vernazza et al., Astron. Astrophys. 451, L43 (2006)

F.J. Vine, D.H. Matthews, Nature 199, 947 (1963)

F.J. Vine, J.T. Wilson, Science 150, 485 (1965)

K. Vozoff, in Electromag. Meth. Appl. Geophys, vol. 2, ed. by M.N. Nabighian (Soc. Explor Geophys., Tulsa, 1991), Part B, p. 641

K. Watanabe, T. Sato, J. Geophys. Res. 95, 75 (1990)

X.-D. Wang et al., Geophys. Res. Lett. 37 (2010). doi:10.1029/2010GL042891

J.M. Weygand et al., J. Geophys. Res. 112 (2007). doi:10.1029/2007JA012486

J.M. Weygand et al., J. Geophys. Res. 114 (2009a). doi:10.1029/2008JA013766

J.M. Weygand et al., EOS Trans. 90 (2009b)

Y.C. Whang, N.F. Ness, J. Geophys. Res. 75, 6002 (1970)

Y.C. Whang, N.F. Ness, J. Geophys. Res. 77, 1109 (1972)

M.A. Wieczorek, B.P. Weiss, Lunar Planet. Inst. Sci. Conf. Abstr. 41, 1625 (2010)

M.A. Wieczorek et al., Rev. Mineral. Geochem. 60, 221 (2006)

P. Wiedelt, J. Geophys. 38, 257 (1972)

M. Wieser et al., Planet. Space Sci. (2009). doi:10.1016/j.pss.2009.09.012

M. Wieser et al., Geophys. Res. Lett. 37 (2010). doi:10.1029/2009GL041721

J.K. Wilson et al., Geophys. Res. Lett. 30 (2003). doi:10.1029/2003GL017443

J.K. Wilson et al., J. Geophys. Res. 111 (2006). doi:10.1029/2005JA011364

P. Wurtz et al., Icarus 191, 486 (2007)

S. Yokota, Y. Saito, Earth Planets Space 57, 281 (2005)

S. Yokota et al., Geophys. Res. Lett. 36 (2009). doi:10.1029/2009GL038185 